\title{
Implications of Gauge-Free Extended Electrodynamics
}

\author{
Donald Reed ${ }^{1}$ and Lee M. Hively ${ }^{2, *}$ \\ 1 State University of New York, Buffalo, NY 14260, USA; torsionpower@yahoo.com \\ 2 University of South Alabama, Mobile, AL 36688, USA \\ * Correspondence: lee.hively314@comcast.net
}

Received: 12 November 2020; Accepted: 12 December 2020; Published: 18 December 2020

\begin{abstract}
Recent tests measured an irrotational (curl-free) magnetic vector potential ( $\mathbf{A})$ that is contrary to classical electrodynamics (CED). A (irrotational) arises in extended electrodynamics (EED) that is derivable from the Stueckelberg Lagrangian. A (irrotational) implies an irrotational (gradient-driven) electrical current density, J. Consequently, EED is gauge-free and provably unique. EED predicts a scalar field that equals the quantity usually set to zero as the Lorenz gauge, making A and the scalar potential $(\Phi)$ independent and physically-measureable fields. EED predicts a scalar-longitudinal wave (SLW) that has an electric field along the direction of propagation together with the scalar field, carrying both energy and momentum. EED also predicts the scalar wave (SW) that carries energy without momentum. EED predicts that the SLW and SW are unconstrained by the skin effect, because neither wave has a magnetic field that generates dissipative eddy currents in electrical conductors. The novel concept of a "gradient-driven" current is a key feature of US Patent 9,306,527 that disclosed antennas for SLW generation and reception. Preliminary experiments have validated the SLW's no-skin-effect constraint as a potential harbinger of new technologies, a possible explanation for poorly understood laboratory and astrophysical phenomena, and a forerunner of paradigm revolutions.
\end{abstract}

Keywords: scalar-longitudinal wave; gradient-driven current; extended electrodynamics

\section{Introduction}

Maxwell's equations are the cornerstone of modern physics, accurately predicting numerous tests and providing the basis for sophisticated 21st century technologies. Classical electrodynamics (CED). is considered to be a firmly established physical theory. Extension of CED to quantum electrodynamics (QED) describes (sub) atomic-scale, matter-light interactions, agreeing with experiments to astounding precision. Thus, mainstream physicists consider CED and QED to be complete and closed with no compelling reason for re-evaluation or alteration. These beliefs arise from the psychological principle of cognitive dissonance that dismisses contrary claims as heretical.

One motivation for this work is that Feynman's 'proof' of Maxell's equations [1] uses assumptions that make CED inconsistent and incomplete (Section 5). Woodside's uniqueness proof [2] of extended electrodynamics (EED) makes no such assumptions, yielding Equations (10)-(14). CED lacks the scalar field (C) that leads to new electrodynamic forces and energy terms in Equations (24) and (25), below. Thus, a second motivation for this work is presentation of first-principles theory and recent test results to warrant a thoughtful re-evaluation of CED's consistency and completeness.

The outline of this paper follows. Section 2 shows that the vector potential ( A) is a measureable, physical quantity via theory and compelling experimental evidence. The curl-free (irrotational) vector potential is important in both classical and quantum domains, for example in the Aharonov-Bohm effect and the Maxwell-Lodge effect. We show that A and scalar-potential $(\Phi)$ have the same physical significance as the electric ( E) and magnetic ( B) fields Section 3 further elucidates $\mathbf{A}$ and $\Phi$, as 
physically measureable and independent fields for EED theory. Section 4 discusses the EED Lagrangian and scalar field $(C)$. [Note that bold font denotes a 3-dimensional vector (e.g., $\mathbf{A}, \mathbf{B}, \mathbf{E}$ ) and italic font denotes scalar quantities (e.g., C)] The centerpiece of this work is Section 5, which presents EED as elucidated by the second author [3]. EED is consistent with preliminary tests [3], showing that an irrotational electrical current drives the scalar longitudinal wave, and includes the missing scalar field for a provably unique EED theory [2]. Section 6 expounds on the SLW's no-skin-effect constraint, revealing vast applications of EED in new technologies and in natural systems. Section 7 explores the possible future role that EED may play in quantum physics and general relativity. Section 8 presents our conclusions with a discussion of future prospects.

\section{Physical Significance of the Magnetic Vector Potential}

Great controversy surrounds the role of the magnetic vector potential in CED [4-6]. This controversy arises from the fundamental mathematical relationship between the potentials ( $\Phi$ and $\mathbf{A})$, and the electric ( $\mathbf{E}$ ) and magnetic ( B) fields. B and $\mathbf{E}$ can be expressed in terms of $\Phi$ and $\mathbf{A}$, as [7]:

$$
\mathbf{B}=\nabla \times \mathbf{A} ; \mathbf{E}=-\nabla \Phi-\frac{\partial \mathbf{A}}{\partial t}
$$

$\mathbf{B}$ and $\mathbf{E}$ are regarded as physical fields; $\Phi$ and $\mathbf{A}$ are viewed as mathematical conveniences for solving Maxwell's equations. This interpretation arises from the detectability of $\mathbf{B}$ and $\mathbf{E}$ through the Lorentz-force equation [7] on a point-particle at the position $\left[\mathbf{r}_{\mathbf{q}}(t)\right]$ with a charge $(q)$ and mass $(M)$ :

$$
\frac{d(M \mathbf{v})}{d t}=q \mathbf{E}+q \mathbf{v} \times \mathbf{B} .
$$

Maxwell's equations are arbitrary (non-unique) in $\Phi$ and $\mathbf{A}$ under the transformation [8]:

$$
\mathbf{A} \rightarrow \mathbf{A}+\nabla \Lambda ; \Phi \rightarrow \Phi-\frac{\partial \Lambda}{\partial t}
$$

The transformation of Equation (3) leaves $\mathbf{B}$ and $\mathbf{E}$ unchanged in Equation (1), and is termed gauge invariance [8]. Equation (3) allows an $\boldsymbol{\aleph}_{2}$-infinitude of choices for $\Lambda$. An analyst can choose an appropriate gauge to fix boundary conditions, find the 'proper' $\mathbf{B}$ and $\mathbf{E}$ fields for known charge and current distributions, or solve an inverse problem. This convention is now engrained in CED, but restricts the potentials. For instance, the Lorenz gauge makes the $\Phi$ and $\mathbf{A}$ inter-dependent.

The physical significance of the electromagnetic potentials has been debated for decades, as discussed further below. Majumdar and Ray $[9,10]$ observe that the $\Phi$ - and A-wave equations are non-invertible despite their gauge ambiguity. These wave equations arise from CED via standard Fourier transforms with a unique projection operator that makes a gauge choice superfluous. Nikolova [11] notes that nonzero, radially-propagating (irrotational) potentials occur in antenna theory with zero Band $\mathbf{E}$-field vectors via expansion of the free-space fields in spherical harmonics. This irrotational "potential wave" is called "longitudinal," meaning A-polarization in the direction of propagation. The corresponding wave impedance is equal to that of free space. CED predicts that this solution has no radiated power, because the Poynting vector is zero. EED predicts a scalar-longitudinal wave (SLW) with a longitudinal- $\mathbf{E}$ field and radiated power (Section 5).

An insightful, under-appreciated dissertation by A. Konopinski [12] elucidates $\mathbf{A}$ and its corresponding physical significance in a form that is quite close to that of Faraday [13-17]. Namely, the vector potential is a "store" of electrodynamic-field momentum available for exchange with the kinetic momentum of charged particles in a conductor [12]. Konopinski shows that operational definitions of $\Phi$ and $\mathbf{A}$ arise from Equation (2), in terms of the potentials in Equation (1):

$$
\frac{d(M \mathbf{v}+q \mathbf{A})}{d t}=q \nabla(\mathbf{v} \bullet \mathbf{A}-\Phi)
$$


This form expresses energy and momentum exchanges without explicit forces. Equation (4) equates the change in "conjugate momentum" ( $\mathbf{p}=M \mathbf{v}+q \mathbf{A})$ to the negative gradient of "interaction energy," $q(\Phi-\mathbf{v} \cdot \mathbf{A})$. Konopinski used a novel gedanken-experiment to show that $\mathbf{A}$ can be measured everywhere in space. Namely, a charged, macroscopic bead slides freely on a circular, non-conductive fiber that is concentric with the cross-section of a magnetic solenoid. A has only an azimuthal component parallel to the current flow, resulting in $\nabla \Phi=\nabla \bullet \mathbf{A}=0$. So, the right-hand side (RHS) of Equation (3) is zero, making the conjugate momentum $(\mathbf{p})$ a conserved quantity. $\mathbf{A}$ is obtained by monitoring the associated change in the bead's kinetic momentum $(M \mathbf{v})$ as the solenoid's current changes. Then, $q \mathbf{A}$ is the conjugate momentum [12] in an external field, just as $q \Phi$ is electrodynamic-field energy per unit charge. These joint properties of the imposed fields arise from their interference and determine the processes, by which fields and charges become observable. Force and power per unit charge are then expressed in terms of the transfer rates.

In addition, much experimental evidence validates the irrotational (curl-free) magnetic vector potential in quantum-, meso-, and macro-scale spatial domains, spanning the full electrodynamic frequency spectrum. For example, the Aharonov-Bohm effect (ABE) $[18,19]$ involves quantum physics of an electrically charged particle ( $q=$ charge) via coupling of the scalar $(\Phi)$ and vector $(\mathbf{A})$ potentials with the complex phase of the particle's wave function. For a particle travelling along a path $(\boldsymbol{P})$ in a region with zero magnetic field ( $\mathbf{B})$ and $\mathbf{A} \neq \mathbf{0}$, the magnetic phase shift is $\varphi=(q / \hbar) \int P \cdot d x \bullet \mathbf{A}$. Here, $\hbar$ is Planck's constant divided by $2 \pi ; \int_{P} d x$ is the path integral along the particle's trajectory. Particles with the same starting and ending points acquire a phase difference $(\Delta \varphi)$ by travelling along two distinct paths around a multiply connected domain:

$$
\Delta \varphi=\frac{q}{\hbar} \oint d x \bullet \mathbf{A}=\frac{q}{\hbar} \int_{\mathbf{S}} \mathbf{d} \mathbf{S} \bullet \nabla \times \mathbf{A}=\frac{q}{\hbar} \int_{\mathbf{S}} \mathbf{d} \mathbf{S} \bullet \mathbf{B}=\frac{q \Phi_{B}}{\hbar}
$$

The second form is the full path around the multiply connected domain. Use of Stoke's theorem yields the third form. Substitution of $\mathbf{B}=\nabla \times \mathbf{A}$ gives the fourth form with $\Phi_{B}$ as the enclosed magnetic flux in the fifth form. The wave nature of quantum particles allows the same particle to traverse different paths. Then, the phase difference is observable by placing a magnetic solenoid between the slits of a double-slit experiment. Tests by Tonomura et al. [20] and Osakabe et al. [21] definitively validated Equation (5). The magnetic phase shift has also been observed in micrometer-sized metal rings [22-24], showing that electrons keep their phase coherence despite diffusion in mesoscopic samples. Wu and Yang [25] proved that $\mathbf{B}$ and $\mathbf{E}$ cannot explain the ABE, which is over-described by the local value of $\mathbf{A}$. Rather, a path-dependent integral over A around multiply-connected, topological regions yields Equation (5) [26].

An electric ABE was also predicted [18,19], depending on a variation in $\Phi$ along two different paths with zero electric field ( $\mathbf{E})$. The phase shift in the wave function is:

$$
\Delta \varphi=-\frac{q \Phi t}{\hbar} .
$$

Here, $t$ is the time spent in the potential. Tests by van Oudenaarden et al. [27] measured quantum interference of electrons in metal rings with two tunnel junctions and a voltage difference between the two ring segments. These tests validated Equations (5) and (6), showing that $\mathbf{A}$ and $\Phi$ play interchangeable roles. Additional experiments are described next.

Varma et al. [28] observed a variable, curl-free vector potential from a magnetic solenoid via a low-current electron beam, propagating linearly along a magnetic field [29]. Contrary to the CED prediction, the current showed time-periodic variations with a linear change in the vector potential [30], implying detection of a macroscale, curl-free A. Varma [31] claimed that this result is mediated by a macroscale, quantum modulation of the de Broglie matter wave along the magnetic field lines, resulting in a scattering-induced transition across electron Landau levels [32]. Shukla [33] commented that the matter wave occurs over centimeters, as a classical effect. Shukla then noted a paradox, namely that a 
curl-free vector potential should not affect a classical system. This apparent paradox is resolved by EED (below).

Experiments by Oliver Lodge used an iron-core, toroidal solenoid, to which an alternating voltage was applied. Lodge measured a voltage in the secondary coil, despite no magnetic field outside the primary coil. The Maxwell-Lodge effect (MLE) is the term for this result, showing that a curl-free vector potential ( A) affects classical electrodynamic systems [34]. Lodge's findings were ignored, and ascribed to deficiencies in the 19th century electrical equipment. Blondel [35] replicated this test. Rousseaux et al. [34] note that the MLE presents a fundamental paradox for CED. Figure 1 shows the test geometry for replication of Lodge's test with modern instrumentation with a long solenoid encircled at its mid-plane by a (secondary) conducting loop [34]. Time-variation in the solenoid's current produces a magnetic field inside the solenoid; no magnetic field occurs outside the solenoid. A is time-varying both inside and outside the solenoid and is parallel to the current. Rousseaux et al. measured a voltage in the secondary coil from $\mathbf{A}$ outside the solenoid via Equation (1), $\mathbf{E}=-\partial$ $\mathbf{A} / \partial t$. Rousseaux et al. [34] note that the gauge indeterminacy in the vector potential is negated by the boundary conditions, showing the physical significance of A.

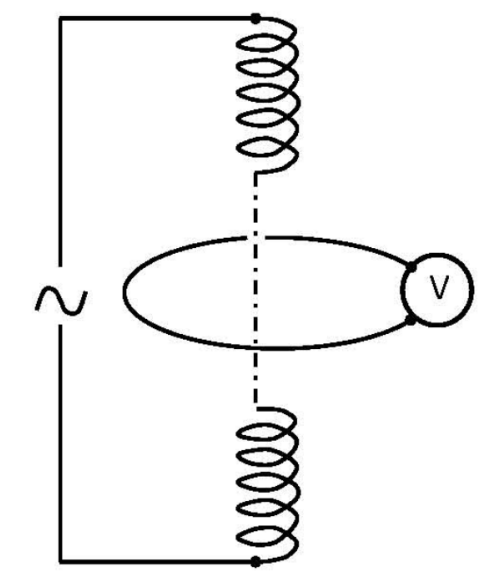

Figure 1. Circuits for the Maxwell-Lodge effect representation [34].

Daibo et al. [36] measured the MLE, using a coiled coil (Figure 2): a very long flexible solenoid whose return-current wire ran through the coil's center to create a pure vector potential with $\mathbf{B}=\mathbf{0}$. Several secondary coils were passed through the hollow core of the vector-potential coil. The coiled coil (primary) was driven with alternating current that induced voltages in the secondary coils that were path independent and occurred, when enclosed by a (super)conducting material. Daibo [37] patented the novel vector-potential transformer (VPT) and related devices, involving high precision measurements in: living organisms (e.g., medical diagnostics), sea water, nuclear-power-plant reactor pressure vessels, and AC electric-field generation without bare electrodes in corrosive media.

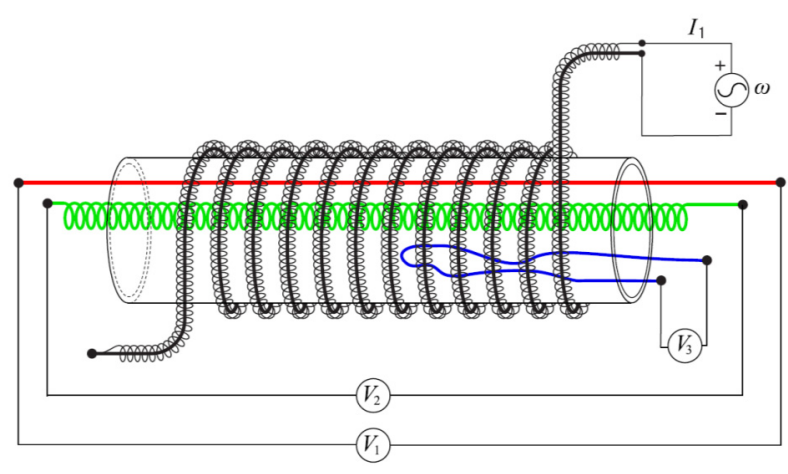

Figure 2. VPT with secondary circuit coil configurations [36]. 


\section{Road to Re-Structure: Independence of Non-Gauged Potentials}

The above theory and experiments provide compelling evidence that the vector potential is a measureable, physical quantity, rather than a mathematical convenience in CED. The CED emphasis on the $\mathbf{E}$ - and B-fields (and their six 3-space components) is then presumptuous and counter-productive, in sharp disagreement with quantum electrodynamics. QED uses the covariant 4-vector potential that describes the effective momentum-energy state of an electromagnetic system (e.g., ABE). The result is contradicting views between CED and QED.

The above experiments expose major flaws in CED under the falsifiability theorem, which states that a theory cannot be proved, but can only be disproved, even by a single, contrary test result. (A 20th century example is the Michelson-Morley experiment that motivated special and general relativity.) Simple, patented devices (e.g., [37]) elucidate low-energy, classical processes that involve the curl-free vector potential in CED. The MLE elevates $\mathbf{A}$ to physically-measureable field in CED. Indeed, Faraday [13-17] and Maxwell [38-40] originally proposed this idea.

We propose two extensions to CED. First, the potential fields ( $\mathbf{A}$ and $\Phi$ ) are physically measureable quantities at all spatio-temporal scales, eliminating the unnatural separation of CED and QED. Second, the potentials are independent of one another, resulting in a provably-unique, gauge-free electrodynamics, as discussed in Section 5. This proposal follows the Occam's razor test, which posits that the simplest model with the fewest assumptions is best. Consequently, we propose an extension of Maxwell's equations without any requirement for gauged potentials. These two extensions of CED imply that the Minkowski electromagnetic 4-potential, $A_{\mu}=(\Phi / c, \mathbf{A})$, is a single unit, as advocated by Majumdar and Ray $[9,10]$. These authors note the absence of a fully (special) relativistically, covariant electrodynamic formulation in many excellent texts, despite its implicit presence in CED as discovered by Einstein in 1905 [41]. Specifically, the CED formulation in terms of the E- and B-fields has no obvious relativistic invariance in different inertial frames. When the equations are written in terms of $\Phi$ and $\mathbf{A}, C E D$ is manifestly invariant under Lorentz boosts and spatial rotations. The latter formulation is therefore preferable for explicit display of the special-relativistic symmetry that is intrinsic to CED.

\section{Derivation of the Form for the Scalar Field}

CED's incompleteness begins with the Helmholtz theorem [7], which states that a smooth 3-dimensional vector field $(\mathbf{U})$ is uniquely decomposable as irrotational $(\nabla V)$ and solenoidal $(\nabla \times \mathbf{W})$ parts: $\mathbf{U}=\nabla V+\nabla \times \mathbf{W}$. Here, $V$ and $\mathbf{W}$ are scalar and vector space-time functions, respectively. Woodside [42,43] proved a unique decomposition of a smooth Minkowski 4-vector field (3 spatial dimensions plus time), into 4-irrotational and 4-solenoidal parts with normal and tangential components on the bounding surface. Woodside [2] then used the Stueckelberg Lagrangian [44]:

$$
\mathfrak{L}=-\frac{\varepsilon c^{2}}{4} F_{\mu v} F^{\mu v}+J_{\mu} A^{\mu}-\frac{\gamma \varepsilon c^{2}}{2}\left(\partial_{\mu} A^{\mu}\right)^{2}-\frac{\varepsilon c^{2} k^{2}}{2}\left(A_{\mu} A^{\mu}\right) .
$$

$F^{\mu v}$ is the Maxwell field tensor; $c$ is the speed of light (not necessarily vacuum); $J_{\mu}=(\rho c, \mathbf{J})$ is the 4-current; the 4-potential is $A_{\mu}=(\Phi / c, \mathbf{A})$; the Compton wave number for a photon with mass $(m)$ is $k=2 \pi m c / h$; and $h$ is Planck's constant. The source terms are the electric charge density $(\rho)$ and the electrical current density ( $\mathbf{J}) ; \varepsilon$ and $\mu$ are the electrical permittivity and magnetic permeability, respectively (not necessarily vacuum). The fully-relativistic Stueckelberg Lagrangian includes both $\mathbf{A}$ and $\Phi$ and resolves many issues with previous CED Lagrangians. For $\gamma=0$ and $m>0$, Equation (7) yields the Maxwell-Proca theory, for which a 2012 test [45] measured $m \leq 10^{-54} \mathrm{~kg}$ (equivalent to $\leq 10^{-18} \mathrm{eV}$ ), consistent with massless photons. For $\gamma=1$ and $m=0$, Equation (7) is [43]:

$$
\mathfrak{L}=\frac{\varepsilon c^{2}}{2}\left[\frac{1}{c^{2}}\left(\nabla \Phi+\frac{\partial \mathbf{A}}{\partial t}\right)^{2}-(\nabla \times \mathbf{A})^{2}\right]-\rho \Phi+\mathbf{J} \bullet \mathbf{A}-\frac{\varepsilon c^{2}}{2}\left(\nabla \bullet \mathbf{A}+\frac{1}{c^{2}} \frac{\partial \Phi}{\partial t}\right)^{2} .
$$


Equation (8) has two classes of 4-vector fields [42]. One has a zero 4-divergence of $A^{\mu}\left(\partial_{\mu} A^{\mu}=0\right)$ and is called 4-solenoidal. The other has zero 4-curl of $A_{\mu}, F^{\mu v}=\partial^{\mu} A^{v}-\partial^{v} A^{\mu}=0$ (four-irrotational vector field), if and only if the last term in Equation (8) is included. The form inside the parentheses of the last term is the Lorenz gauge when set to zero [8], restricting $\Phi$ and $\mathbf{A}$ to be dependent on each other. However, EED allows for a non-zero value of this scalar expression, making the potentials completely independent of one another. The scalar field arises from the third term on the right-hand side of Equation (7) for $\gamma=1$ and $m=0$. Equation (8) shows clearly how this term arises by writing the Lagrangian density in terms of $\Phi$ and $\mathbf{A}$ for a massless 4-vector field $\left(A^{\mu}\right)$. Thus, the relationship between $\Phi$ and A reveals the missing scalar field and its key role in EED. The result is a provably-unique, gauge-free formulation of extended electrodynamics (EED) with a new scalar component $(C)$ as a space-time function [2]:

$$
C=\nabla \bullet \mathbf{A}+\frac{1}{c^{2}} \frac{\partial \Phi}{\partial t}
$$

Application of the Lorenz gauge $(C=0)$ eliminates the physical meaning of the scalar field. However, the independence of the potentials in gauge-free EED predicts the scalar field $(C)$, giving rise to the unique dynamics of the scalar-longitudinal wave and the scalar wave (Section 5).

Additional papers have appeared over the the last 20 years. Jack [46] used a quaternion formalism to show that the scalar ("temporal") field can explain thermoelectricity. Saa $[47,48]$ showed that CED fails to reveal a Doppler shift, when a boost is produced in the direction of motion; a Doppler shift in $\mathbf{B}$ and $\mathbf{E}$ does arise when a scalar field is incorporated in the model. Williamson [49-52] used Clifford algebra to formulate an extended model of elementary particles with the scalar field and a topological structure for photons and electron-positron pair production/annihilation.

Other work examined extended electrodynamics or the effects of relaxing the Lorenz gauge: Fock and Podolsky [53] in 1932; Dirac, Fock, and Podolsky [54] in 1932; Stueckelberg [44] in 1938; Ohmura [55] in 1956; Milner [56] in 1960; Aharonov and Bohm [57] in 1963; Alicki [58] in 1978; Cornille [59,60] in 1990-1994; Munz et al. [61] in 1999; Woodside [2,42,43] in 1999-2009; van Vlaederen [62,63] in 2001-2003; Arbab [64,65] in 2009 and 2018; Jimenez and Maroto [66] in 2011; Hively and Giakos [67] in 2012; Hively [68] in 2016; Modanese [69-78] in 2016-2019; Islamov and Tomilin [79] in 2017; Banduric [80] in 2017; Celani et al. [81] in 2017; Hively and Loebl [3] in 2019; Keller and Hively [82,83] in 2019; and Haralick [84] in 2018. Other recent works are relevant [85-89]. This paragraph and the previous paragraph cite 30 independent works that are indicative of the importance of EED.

\section{EED and the Scalar Lonitudinal Wave}

This section focuses on the EED formulation $[3,67]$ that rigorously extends Woodside's work [2,42, 43]. EED's dynamical equations can be derived from the Lagrangian in Equation (8):

$$
\begin{gathered}
\mathbf{E}=-\nabla \Phi-\frac{\partial \mathbf{A}}{\partial t} ; \\
\mathbf{B}=\nabla \times \mathbf{A} ; \\
C=\nabla \bullet \mathbf{A}+\frac{1}{c^{2}} \frac{\partial \Phi}{\partial t} ; \\
\nabla \times \mathbf{B}-\frac{1}{c^{2}} \frac{\partial \mathbf{E}}{\partial t}-\nabla C=\mu \mathbf{J} ; \\
\nabla \bullet \mathbf{E}+\frac{\partial C}{\partial t}=\frac{\rho}{\varepsilon} .
\end{gathered}
$$

Woodside [2] proved the uniqueness of Equations (10)-(14). Equation (8) implies Equations (10)-(14) [42]. Moreover, Equations (10)-(14) imply Equation (8) [2,42,43]. Equation (8) is then necessary and sufficient for Equations (10)-(14). The Lagrangian for curved space-time [66] reduces to Equation (8) in Minkowski 
4-space. Equation (10) is equivalent to Faraday's law; Equation (11) is equivalent to the no magneticmonopoles equation.

EED has a new term $(-\nabla C)$ in Ampere's Law (Equation (13)), and a new term $(\partial C / \partial t)$ in Gauss' Law (Equation (14)). As before, $\rho$ is the charge density, and $\mathbf{J}$ is the current density vector. Equation (13) explicitly decomposes $\mathbf{J}$ into solenoidal $(\nabla \times \mathbf{B})$ and irrotational $(\nabla C)$ parts, in accord with the Helmholtz theorem. The new terms in Equations (13) and (14) change only the irrotational (longitudinal) electrodynamics, as elucidated by Keller and Hively $[82,83]$. These irrotational components sre gauged away in CED by Equation (3). Thus, the solenoidal (transverse) electrodynamics remain unchanged, as described by CED.

Equations (10)-(14) have important implications [3,67]. First, (special) relativistic covariance is preserved. Second, the $\mathbf{E}$ - and $\mathbf{B}$-fields are unchanged in terms of the classical potentials ( $\mathbf{A}$ and $\Phi$ ) in Equation (1). Third, EED yields the same wave equations for $\mathbf{E}$ and $\mathbf{B}$ as CED. Fourth, the $\mathbf{A}$-wave equation is obtained by replacing $\mathbf{B}, C$, and $\mathbf{E}$ in Equation (13) with Equations (10)-(12); using the vector calculus identity [90], $\nabla \times \nabla \times \mathbf{A}=\nabla(\nabla \bullet \mathbf{A})-\nabla^{2} \mathbf{A}$; and noting that $\partial \nabla C / \partial t-\nabla \partial C / \partial t=0$. Fifth, the $\Phi$-wave equation arises by: substituting $\mathbf{E}$ and $C$ from Equations (10) and (12) into Equation (14); and noting that $\partial \nabla \bullet \mathbf{A} / \partial t-\nabla \bullet \partial \mathbf{A} / \partial t=0$. Sixth, the $\mathbf{A}$ - and $\Phi$-wave equations are derived without a gauge condition, making EED gauge free. Seventh, EED predicts a scalar-longitudinal wave (SLW), consisting of a E-field along the direction of propagation (longitudinal) and the scalar field (C); the SLW carries energy and momentum. Eighth, EED predicts a scalar wave (SW) that carries only energy. Ninth, both the SLW and SW are unconstrained by the skin effect, because they lack a B-field that induces eddy currents. Tenth, recent SLW tests [3] are consistent with previous work by Tesla [91], Monstein and Wesley [92], and Meyl [93]. Eleventh, the A-, B-, C-, E- and $\Phi$-wave equations are unchanged under time reversal, so that reciprocity holds. A SLW transmitter can then be used as a receiver. Twelfth, EED predicts relations among the longitudinal field components:

$$
\mathbf{A}^{L}=\nabla \alpha \Leftrightarrow \mathbf{E}^{L}=\nabla e \Leftrightarrow \mathbf{J}^{L}=\nabla \kappa .
$$

The superscript $(L)$ denotes the longitudinal component; $\alpha, e$, and $k$ are scalar, space-time functions. Equation (15) implies zero magnetic field ( $\left.\mathbf{B}=\nabla \times \mathbf{A}^{L}=\nabla \times \nabla \alpha=\mathbf{0}\right)$ and vice versa.

EED predicts a $C$-wave equation from the divergence of Equation (13); use of $\varepsilon \mu(\partial / \partial t)$ on Equation (14); and summing the two results with $\nabla \bullet \nabla \times \mathbf{B}=0$ [90]:

$$
\frac{\partial^{2} C}{\partial c^{2} t^{2}}-\nabla^{2} C \equiv \square^{2} C=\mu\left(\frac{\partial \rho}{\partial t}+\nabla \bullet \mathbf{J}\right)
$$

Equation (16) is local in space and time. However, all experiments are performed over a finite time, $\Delta T$, i.e., a time average. A long-time average gives $\partial \rho / \partial t+\nabla \bullet \mathbf{J}=0$ on the right-hand side (RHS) of Equation (16), in accord with long-standing experiments that validate classical charge balance [94]. The lower bound on electron lifetime for charge balance has been measured as $\geq 6.6 \times 10^{28}$ years [95] (decay into two $\gamma$-rays, each at with an energy of $m_{e} c^{2} / 2=0.256 \mathrm{MeV} ; m_{e}=$ electron mass). Nevertheless, long-time charge conservation is not inconsistent with charge non-conservation over short-time scales, $\Delta T \leq \Delta t$, per the Heisenberg uncertainty relation, $\Delta E \Delta t \geq \hbar / 2$. Here, $\Delta E$ is the charged-quantum-fluctuation energy; and $h$ is Planck's constant divided by $2 \pi$. Equation (16) can be interpreted as charge non-conservation (particle-antiparticle fluctuations [PAPF]) driving $C$, and vice versa, not unlike energy-fluctuations driving mass-fluctuations in quantum theory and vice versa $[3,67]$. Equivalently, the RHS can be interpreted as PAPF over some non-local region $(\Delta x)$, consistent with the Heisenberg uncertainty relation, $\Delta p \Delta x \geq \hbar / 2$. Here, $\Delta p$ is the charged-quantum-fluctuation momentum over the non-local region, $\Delta x$. (While this interpretation is novel, it is consistent with PAPF according to the Heisenberg uncertainty principle.) Thus, Equation (16) predicts charge conservation on long time-scales (consistent with $(E D)$, and exchange of energy between $C$ and quantum fluctuations for $\Delta T \leq \Delta t$. Confirmation of these quantum charge fluctuations involves tests, consistent with the Heisenberg uncertainty relation. 
One possible test could use the electron $\left[\Delta E\right.$ (electron) $\left.=m_{e} c^{2}=0.511 \mathrm{MeV}\right]$ corresponding to a time, $\Delta t \sim 6 \times 10^{-22} \mathrm{~s}$. Subzeptosecond dynamics have been measured [96], so a direct measurement of this prediction is feasible. Moreover, quantum fluctuations can control charge quantization [97], in accord with Equation (16).

The interface matching condition for Equation (16) uses a Gaussian pill box with the end faces parallel to the interface in regions " 1 " and " 2 ". Noting that $\nabla^{2} C=\nabla \bullet \nabla C$ and using the divergence theorem in the limit of zero pill-box height yields continuity in the normal component (" $n$ ") of $\nabla C / \mu$ for long times $[3,67]$ :

$$
\left(\frac{\nabla C}{\mu}\right)_{1 n}=\left(\frac{\nabla C}{\mu}\right)_{2 n}
$$

The subscripts, " 1 " and "2" denote medium 1 and medium 2 for $\mu$ not necessarily in vacuum. EED predicts that the interface matching conditions for the $\Phi$ - and $\mathbf{A}$-wave equations are $[3,67]$ :

$$
\begin{gathered}
-(\varepsilon \nabla \Phi)_{2 n}+(\varepsilon \nabla \Phi)_{1 n}=\rho_{A} ; \\
-\left[\frac{(\widehat{\mathbf{n} \bullet \nabla) \mathbf{A}}}{\mu}\right]_{1}+\left[\frac{(\widehat{\mathbf{n} \bullet \nabla) \mathbf{A}}}{\mu}\right]_{2}=\mathbf{J}_{A} .
\end{gathered}
$$

The subscript, " $n$ ", denotes the normal component; the subscripts " 1 " and " 2 " identify the two media. $\quad \mathbf{J}_{A}$ and $\rho_{\mathbf{A}}$ are the interface surface-current density and surface-charge density, respectively; $\widehat{\mathbf{n}}$ is the unit-vector that is normal to the interface; $\varepsilon$ and $\mu$ are the permittivity and permeability, respectively (not necessarily vacuum). Validation tests are clearly needed for these predictions that are inconsistent with CED. CED and EED have the same tangential interface matching conditions.

The RHS of Equation (16) is zero on classical time-scales, as discussed above. The lowest-order solution to the homogeneous $C$-wave equation in spherical coordinates is then given by [8]:

$$
C=\frac{C_{o} \exp [j(k r-\omega t)]}{r} .
$$

Here, $C_{o}$ is the scalar field amplitude; $j=\sqrt{ }-1$; the wave number $k$ is $(2 \pi / \lambda)$ for a wavelength $(\lambda)$; $\omega=2 \pi f$ for a frequency $(f)$; and $r$ is the spherical radius. Boundary conditions for Equation (20), include $C(r \rightarrow \infty) \rightarrow 0$, which is trivially satisfied. Equation (25) predicts that the energy density of the $C$-field is $\left(C^{2} / 2 \mu\right)$, yielding a constant energy, $4 \pi r^{2}\left(C^{2} / 2 \mu\right)$, through a spherical boundary around a source in arbitrary media, as required $[3,67]$. The scalar field then can be interpreted as an electrodynamic pressure, similar to that in acoustics and hydrodynamics, which expands and contracts radially. (By contrast, CED forbids a spherically symmetric transverse wave.) Substitution of Equation (15) into Equation (13) yields a separation into transverse $(T)$ and longitudinal $(L)$ forms via Equations (21a) and (21b), respectively:

$$
\begin{gathered}
\nabla \times \mathbf{B}^{T}-\frac{1}{c^{2}} \frac{\partial \mathbf{E}^{T}}{\partial t}=\mu \nabla \times \mathbf{G}^{T} \Rightarrow \nabla \times\left(\mathbf{B}^{T}-\mu \mathbf{G}^{T}\right)+\frac{\partial^{2} \mathbf{A}^{T}}{\partial c^{2} t^{2}}=0 ; \\
-\frac{1}{c^{2}} \frac{\partial \mathbf{E}^{L}}{\partial t}-\nabla C=\mu \nabla \kappa \Rightarrow \frac{\partial^{2} \mathbf{A}^{L}}{\partial c^{2} t^{2}}=\nabla\left(-\frac{1}{c^{2}} \frac{\partial \Phi}{\partial t}+C+\mu \kappa\right) .
\end{gathered}
$$

$C, \mathbf{E}^{L}$, and $\mathbf{J}^{L}$ are related by Equation (21b) with [98] $\sigma=\varepsilon_{0} \varepsilon^{\prime \prime} \omega$ and $\varepsilon=\varepsilon_{\mathrm{o}}\left(\varepsilon^{\prime}-j \varepsilon^{\prime \prime}\right)$ for a SLW impedance, $Z$ :

$$
\begin{gathered}
Z=\frac{\left|\mathbf{E}_{r}\right|}{\left|C / \mu_{o} \mu^{\prime}\right|}=\sqrt{\frac{\mu_{o}}{\varepsilon_{0}}} \sqrt{\frac{\mu^{\prime}}{\varepsilon^{\prime}}} \frac{1-1 /(j k r)}{1-j \tan \left(\delta_{\varepsilon}\right)} \\
\mathbf{E}^{L}=\frac{E_{r} \hat{\mathbf{r}} \exp [j(k r-\omega t)]}{r} .
\end{gathered}
$$


Equation (22) assumes spherical waves in linearly conductive media, $\mathbf{E}^{L}=\mathbf{J}^{L} / \sigma=\nabla \mathrm{k} / \sigma$, where $\sigma$ is the electrical conductivity. The unit vector in the radial direction is $\hat{\mathbf{r}} ; \varepsilon_{0}$ and $\mu_{0}$ are the free-space permittivity and permeability, respectively; $\varepsilon^{\prime}$ and $\mu^{\prime}$ are the relative permittivity and permeability (not necessarily vacuum), respectively; $\tan \left(\delta_{\varepsilon}\right)=\varepsilon^{\prime \prime} / \varepsilon^{\prime}$. Here, the same definitions are used for $k, r, t$, and $\omega$ as above. From Equation (13), $C$ has the same dimensions as $\mathbf{B}=\mu \mathbf{H}$. Consequently, Equation (22a) uses $|\mathbf{E}| /(C / \mu)$ to obtain the SLW impedance, like the CED form, $Z=|\mathbf{E}| / \mathbf{H} \mid$. The $C$ - and $\mathbf{E}^{L}$-field energies from Equation (25), $4 \pi r^{2}\left(\varepsilon \mathrm{E}^{2} / 2\right)$ and $4 \pi r^{2}\left(C^{2} / 2 \mu\right)$, are constant through a spherical boundary and $C(r \rightarrow \infty) \rightarrow 0$ and $\left|E^{L}(r \rightarrow \infty)\right| \rightarrow 0$. Equation (22a) predicts $Z=\sqrt{ } \mu_{0} / \varepsilon_{0}$ in free-space $\left(\varepsilon^{\prime}=\mu^{\prime}=1\right.$ and $\left.\varepsilon^{\prime \prime}=\mu^{\prime \prime}=0\right)$. The SLW then consists of $C$ and $\mathbf{E}^{L}$ propagating together. A SLW monopole antenna radiates power $\left(\mathbf{P}_{\text {OUT }}\right)$ isotopically in free space [3]:

$$
\mathbf{P}_{\text {OUT }}=\frac{I^{2} \hat{\mathbf{r}}}{(4 \pi r)^{2}} \sqrt{\frac{\mu}{\varepsilon}}
$$

The SLW has not been previously observed, because transverse electromagnetic (TEM) antennas detect only waves that produce a circulating current. A TEM transmitter produces only circulating currents, yielding $C=0$ and $\mathbf{E}^{L}=\mathbf{0}$ with no SLW power output or reception.

The SLW has no magnetic field $(\partial \mathbf{B} / \partial t=\mathbf{0})$, implying no (circulating) eddy currents from Faraday's law. Thus, EED predicts that the SLW is unconstrained by the skin effect. Preliminary experimental results [3] are consistent with this prediction (propagation through thousands of skin-depths of solid copper), using standard electronic instrumentation. This no-skin-effect feature cannot be explained by CED, and has practical applications in wireless communications and wireless transmission of electrical power.

Figure 3 summarizes the key differences between CED versus EED. CED has an inter-dependence of the scalar and vector potentials, solenoidal (circulating currents), and the transverse electromagnetic waves. EED is gauge-free with scalar and vector potentials that are independent of one another, producing the SLW and SW. The VPT is one practical innovation that exploits the SLW and its novel no-skin-effect feature.

\section{Comparison: EED Versus CED (Classical Electrodynamics)}

\section{EED(Non-Zero Div. of Pot.)}

- Scalar-Vector Potentials Independent (not subject to Lorenz Condition) $\downarrow$

- Magnetic Field Zero - Current not subject to Lenz's Law $(\nabla \times \mathbf{A}=0)$ $\downarrow$

- Gradient-Driven Current $(\nabla \times \mathbf{J}=0)$ $\downarrow$

- Scalar Longitudinal Wave
CED (Solenoidal Div. of Pot.)

- Scalar-Vector Potentials Dependent (Lorenz Condition Holds) $\downarrow$

- Magnetic Field Exists - Current subject to Lenz's Law (eddy currents exist). $\downarrow$

- Solenoidal (Circulating) Currents $\downarrow$

- Transverse Electromagnetic Wave

Figure 3. Comparison between EED and CED.

EED includes the transverse (solenoidal) components of the fields $\left(\mathbf{A}^{T}, \mathbf{B}\right.$, and $\mathbf{E}^{T}$ ) in the same way as CED, while adding longitudinal (irrotational) components $\left(\mathbf{A}^{L}, \mathbf{E}^{L}\right.$, and $\left.\mathbf{J}^{L}\right)$ and the scalar field that CED gauges away. Table 1 shows the specific, quantifiable EED predictions, which are discussed next in an item-by item fashion. Items 1-9 involve the scalar-longitudinal wave (SLW, also called the longitudinal-scalar wave or electroscalar wave). The SLW driver is an irrotational current $\left(\mathbf{J}^{L}\right.$, Item 1 in Table 1), or an irrotational vector potential $\left(\mathbf{A}^{L}\right)$, or an irrotational electric field $\left(\mathbf{E}^{L}\right)$; such a driver creates no magnetic field ( $\mathbf{B}=\mathbf{0}$ ). The SLW consists of a scalar field $(C)$-Item 2, together with 
an irrotational electric field ( $\left.\mathbf{E}^{L}\right)$-Item 3. The corresponding SLW power $(C \mathbf{E} / \mu$.) and momentum densities ( $-C \mathbf{E} / \mu$.) -Items $4-5$ are comparable to a transverse electromagnetic (TEM) wave-Item 6. The SLW is unconstrained by the classical skin effect-Item 7, and has a free-space attenuation over distance $(r)$ as $1 / r^{2}$-Item 8 . SLW creation by a monopole transmitter is isotropic-Item 9 . The equation numbers in Table 1 correspond to the equations in this paper, as adapted from [3]. The scalar wave (SW) arises from the conditions of Item 1 with the additional constraint of Item 10. The SW has pressure $\left(\nabla C^{2} / 2 \mu\right)$ and energy $\left(C^{2} / 2 \mu\right)$ densities-Items $11-12$. The scalar wave/field is a pseudo-scalar-Item 13 and can be driven by charge fluctuations (e.g., particle-antiparticle fluctuations)—Item 14. CED cannot explain the SLW/SW, because the longitudinal components $\left(\mathbf{A}^{L}, \mathbf{E}^{L}\right.$, and $\mathbf{J}^{L}$ ) are gauged away. EED predicts a material-interface matching condition for $C$-Item 15 , for the normal component of an electric field from a surface charge density-Item 16, and for the normal component of a vector potential from a surface current-Item 17. Item 15 is new; Items 16-17 are inconsistent with CED. EED also predicts a new, mixed mode force density-Item 18, a new longitudinal force-Item 19, and a new power source term-Item 20 . These EED predictions clearly need careful validation.

Table 1. Summary of testable EED predictions.

\begin{tabular}{|c|c|c|}
\hline Item & Brief Description of Testable Prediction & Reference \\
\hline 1 & The SLW drivers are: $\mathbf{A}^{L}=\nabla \alpha \Leftrightarrow \mathbf{E}^{L}=\nabla e \Leftrightarrow \mathbf{J}^{L}=\nabla \kappa \Leftrightarrow \mathbf{B}=\mathbf{0}$. & Equation (15)* \\
\hline 2 & The SLW has a dynamical scalar field, $C=\nabla \cdot \mathbf{A}+\varepsilon \mu \partial \Phi / \partial t$. & Equation (12) \\
\hline 3 & The SLW has a longitudinal E-field. & Equation $(22 b)$ \\
\hline 4 & The SLW has a power density vector of $C \mathbf{E} / \mu$. & Equation (24) \\
\hline 5 & The SLW has a momentum density of $-C \mathbf{E} / \mu$. & Equation (25) \\
\hline 6 & The SLW has a power comparable to the TEM wave. & Equation $(22 a) *$ \\
\hline 7 & The SLW is unconstrained by the skin effect. & Section $5 *$ \\
\hline 8 & The SLW free-space attenuation goes like $1 / r^{2}$. & Equation $(23) *$ \\
\hline 9 & The SLW monopole radiation is isotropic. & Equation $(23) *$ \\
\hline 10 & The scalar wave arises from $\Phi=-\partial \alpha / \partial t$, plus Item 1 & [3] \\
\hline 11 & The scalar-wave has a pressure density of $\nabla C^{2} / 2 \mu$. & Equation (25) \\
\hline 12 & The scalar-wave has an energy density of $C^{2} / 2 \mu$. & Equation (24) \\
\hline 13 & $C$ is a pseudo-scalar. & Equation (12) \\
\hline 14 & The scalar wave is also charge-fluctuation driven. & Equation (16) \\
\hline 15 & The interface matching condition for $C$ is ... & Equation (17) \\
\hline 16 & The interface matching condition for $\rho_{\mathrm{A}}$ is $\ldots$ & Equation (18) \\
\hline 17 & The interface matching condition for $\mathbf{J}_{A}$ is ... & Equation (19) \\
\hline 18 & Momentum balance has a mixed-mode term, $\nabla \times \mathrm{BC} / \mu$. & Equation (25) \\
\hline 19 & Momentum balance also has source term, $\mathbf{J C}$. & Equation (25) \\
\hline 20 & Energy balance has a new source, $\rho C / \varepsilon \mu$. & Equation (24) \\
\hline
\end{tabular}

The SLW is transmitted (and received) by a novel antenna [68], as shown in Figure 4 (left) [cross-sectional view of a linear, monopole with the coaxial center conductor (202) as the radiator]. The outer conductor (204) is electrically connected to the top of the skirt balun (206) with a length $(\lambda / 4)$ that causes a phase shift in the current from the bottom (inside surface) of $206\left(0^{\circ}\right)$ to the top (inside surface) of $206\left(90^{\circ}\right)$ and back down $204\left(180^{\circ}\right)$. The $180^{\circ}$-phase shift cancels the return current along 204 to form a monopole antenna. Essentially all of the current then goes into charging and discharging the center conductor (202) without creating a displacement field. The resultant current is irrotational, 
which is a necessary and sufficient condition for producing the SLW, The irrotational current does not create a magnetic field.
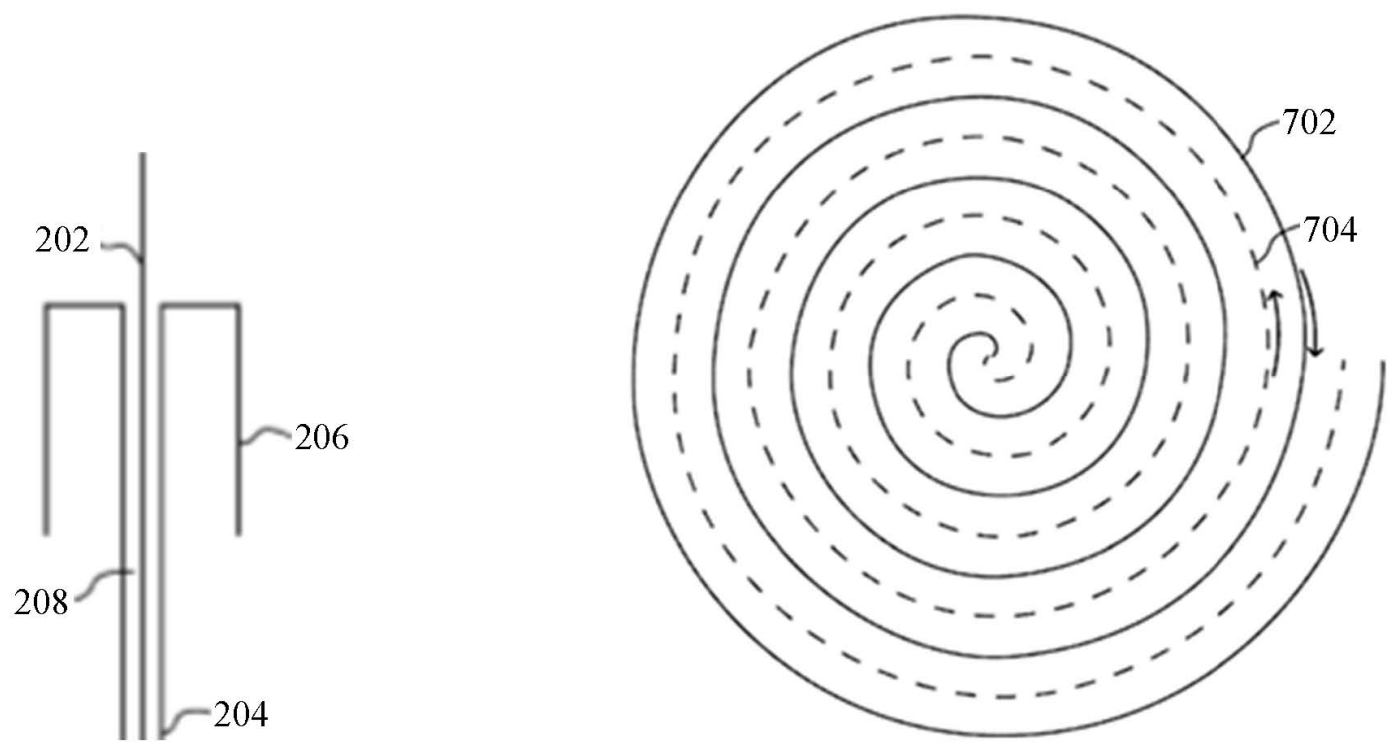

Figure 4. Cross-section of SLW antenna (left) and bifilar-coil-type SLW antenna (right) [68].

Figure 4 (right) shows an alternate antenna design [68], consisting of two spiral conductors that are connected at the coil's center, causing current-flow in opposite directions in adjacent turns. These opposing-current-conductors are represented by the solid (702) and dashed (704) lines. The opposing currents cancel the magnetic field $(\mathbf{B}=\mathbf{0})$, generating of an irrotational current for transmission (and reception) of the SLW. This coil has zero inductance (counter-going electrical currents in adjacent turns), zero capacitance (same electric charge density in adjacent turns), as a 2-dimensional monopole that accumulates positive and negative charge over each half of a sinusoidal cycle for a coil length $<\lambda / 10$. The antennas in Figure 4 are significant, because CED cannot explain the SLW.

\section{Evidence for the SLW: Both Inanimate and Biological}

The purpose of this section is to show experimental evidence that is consistent with EED predictions. These tests (in no particular order) include geophysical SLWs for earthquake prediction [99]; observation of solar-generated SLWs [100]; laboratory tests by Graneau and Hering [101]; X-ray emission during tape peeling [102,103]; irrotational currents in living processes and their implication for biomedical diagnostics and therapeutics [104-107]; and radioactive decay rate variation that is well-correlated with solar time scales [108-113]. These observations cannot be explained by CED. CED is empirically-based and cannot be proved. Rather, it can be falsified, even by a single test. Here, we show several more tests that falsify CED.

Larry Park's invention [99] detects earthquake precursors as single-phase impulses and multiple-phase bursts, hours to days before a seismic event. These precursors are created by rock fractures (breaking of chemical bonds), leaving positive and negative charges on opposite sides of the fault. Eventually, charge accumulation causes arcing across the fault; this gradient-driven current creates the SLW. The precursors are high-frequency pulses superimposed on a broad-band spectrum (mostly low-frequency) spectrum. Figures 26-29 of [99] show counter-going currents in the sensor coil, not unlike Figure 4 (right) above. These counter-going currents enable reception of SLWs. This work clearly needs replication.

NASA has observed solar, breathing-mode, plasma oscillations with ions moving radially outward (inward), while electrons move radially inward (outward). Momentum conservation causes the amplitude and velocity of the ions to be smaller than the electrons by a factor of the square-root of their 
mass ratio, causing a net radial (irrotational) current that creates the SLW. NASA also observed higher frequency, radial, multipole oscillations that create SLWs in the same way. Russian researchers realized that a solar eclipse was favorable for detection of solar-generated SLWs [100]. Namely, a solar eclipse by the moon blocks solar TEM waves, allowing SLWs with much greater penetration power to dominate. Moreover, EED predicts SLW-excited radial oscillations in conductive substances (e.g., a charged sphere acting as SLW receiving antenna). Time-invariance of EED (discussed above) provides reciprocity (e.g., oscillation of a charged sphere acting as a SLW transmitter). Using this physics, the Russian test used four, solid-copper spheres that were connected to each others' centers by a copper wire. The spheres were placed inside a sealed metallic box (Faraday cage) to eliminate TEM radiation. Figure 5 shows the voltage measurement with maximal detection of SLW radiation during the eclipse's totality with the moon's outer shell acting as a spherical lense. SLW radiation should dominate anytime the moon is between the Earth and the sun (monthly new-moon phase); the Russian team [100] also observed this result. These results cannot be explained by CED and clearly need replication.

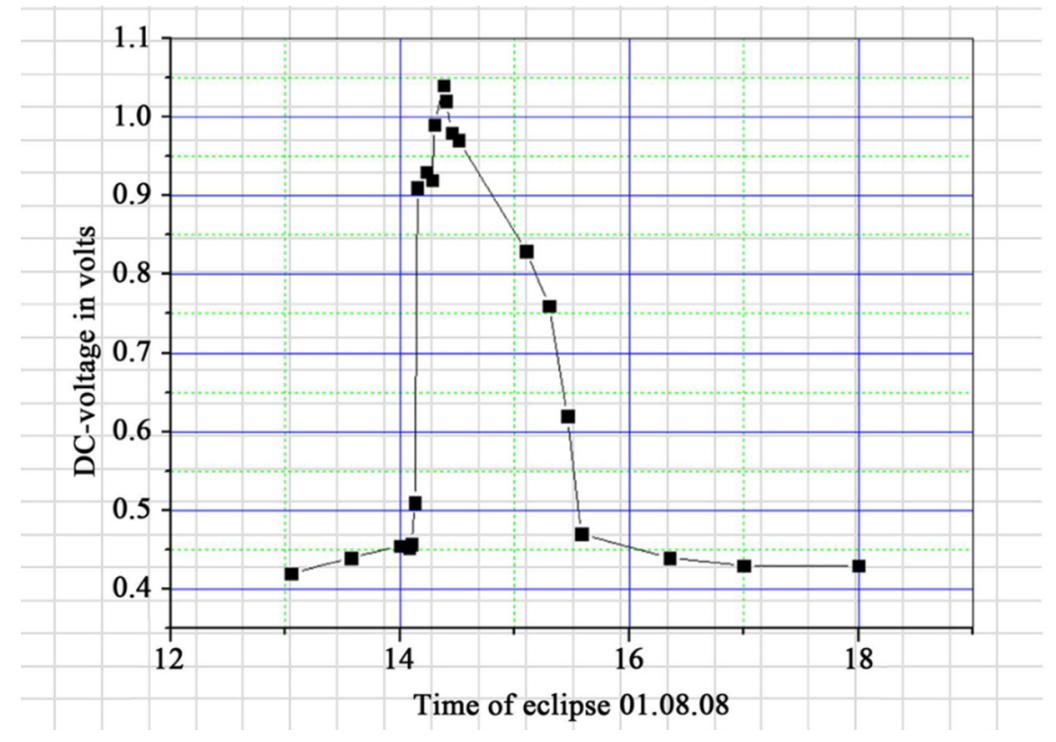

Figure 5. Possible registration of solar SLW radiation [100].

This Russian test raises the possibility of electrical power generation from solar SLWs, as follows. The sun and other stars are spheres of charged particles (electrons and ions, as a high-temperature plasma) that undergo radial oscillations, which in turn produce a radial electrical current, as discussed above. This prospect uses EED's prediction of new terms in the energy balance equation $[3,62,63,67,68]$, Equation (24): scalar field energy $\left(C^{2} / 2 \mu\right)$, SLW energy $(C \mathbf{E} / \mu)$, and a power source $(\rho C / \varepsilon \mu)$.

$$
\frac{\partial}{\partial t}\left(\frac{\mathbf{B}^{2}}{2 \mu}+\frac{C^{2}}{2 \mu}+\frac{\varepsilon \mathbf{E}^{2}}{2}\right)+\nabla \bullet\left(\frac{\mathbf{E} \times \mathbf{B}}{\mu}+\frac{C \mathbf{E}}{\mu}\right)+\mathbf{J} \bullet \mathbf{E}=\frac{\rho C}{\varepsilon \mu} .
$$

New terms occur in the EED momentum balance equation $[3,62,63,67,68]$, Equation (25): SLW momentum flux $(C \mathrm{E} / \mu)$, a mixed mode flux $[(\nabla \times \mathbf{B} C) / \mu]$, a force $(\mathrm{JC})$ parallel to the current density, and scalar-field pressure $\left(\nabla C^{2} / 2 \mu\right)$. The right-most term in Equation (25) is the divergence of the CED Maxwell-stress tensor.

$$
\varepsilon \mu \frac{\partial}{\partial t}\left(\frac{\mathbf{E} \times \mathbf{B}}{\mu}-\frac{C \mathbf{E}}{\mu}\right)+\rho \mathbf{E}+\mathbf{J} \times \mathbf{B}+\frac{\nabla \times \mathbf{B} C}{\mu}=\mathbf{J} C+\frac{\nabla C^{2}}{2 \mu}+\nabla \bullet \overline{\mathbf{T}} .
$$

Equation (25) predicts an additional force $(\mathbf{J C})$ that is parallel to the current density $(\mathbf{J})$ and

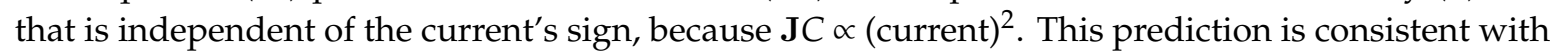
tests [101]: a force on Ampere's bridge, tension that ruptures current-carrying wires, the Graneau-Hering 
submarine, Hering's mercury pump, and an oscillating force in a current-carrying mercury wedge. These tests need replication.

Equation (25) also predicts a momentum loss $(-C \mathbf{E} / \mu)$ with concomitant power gain $(+C \mathbf{E} / \mu)$ in Equation (24), and vice versa. This sign difference means that SLW emission (energy loss) drives a momentum gain in a massive object that emits the SLW.

A 2008 test [102] showed X-ray emission via tribo-electrification by peeling adhesive tape, which breaks adhesive (chemical) bonds, causing charge separation that stores electrostatic energy at thousands of volts. The subsequent electrical-arc discharge accelerates electrons that are absorbed on the other side of the interface, producing Bremstrahlung ("braking radiation" as the electrons abruptly decelerate). CED predicts transverse Bremstrahlung. However, the transient electron-charge density of $10^{12}$ electrons $/ \mathrm{cm}^{2}$ was more than ten-fold greater than typical tribo-charging systems. A 2010 experiment measured the angular distribution of X-rays during tape peeling [103] that is inconsistent with the CED prediction for transverse Bremsstrahlung (Figure 6). Specifically, ordinary Bremsstrahlung bounds the angular distribution from below, while polarizational Bremsstrahlung bounds the angular distribution from above. Neither model predicts the $20 \%$ peak between $80^{\circ}$ to $100^{\circ}$. EED predicts that the SLW is unconstrained by the skin effect and is more penetrating in matter. We conjecture that the SLW-Bremsstrahlung might explain the angular distribution in Figure 6, which CED cannot explain. Careful replication tests are needed.

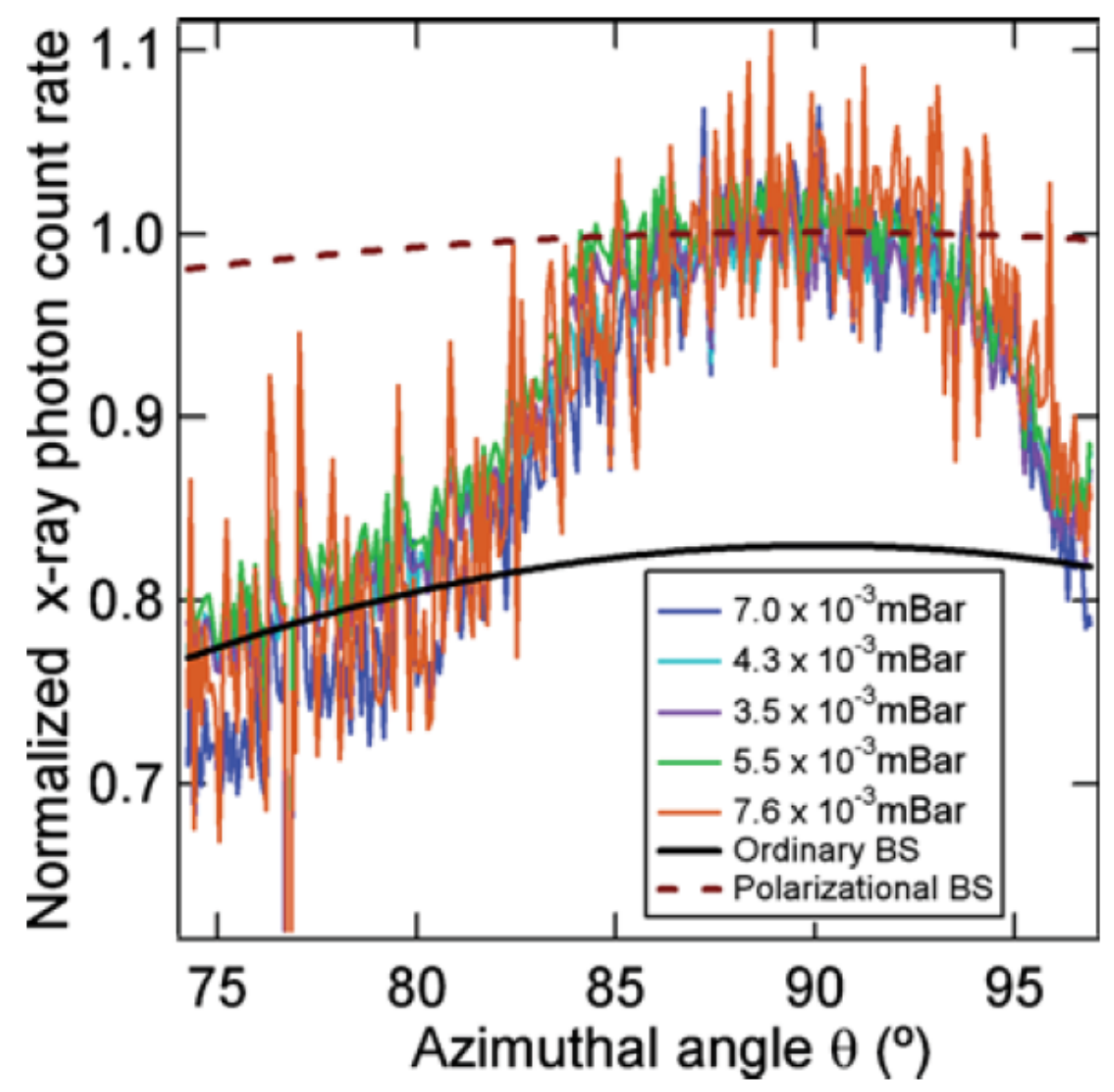

Figure 6. Spectral output of radiation as a function of tape angle [103].

All living processes involve ion-concentration, gradient-driven currents across cell membranes [104]. These currents are the basis for electro-physiological, medical diagnostics and treatments. For example, scalp brain-waves arise from irrotational currents [105]. Thus, SLW-irradiation of living cells will alter 
these currents. The TELSAR watch [106] has measureable biological effects due to very low frequency SLWs. Krasnoholovets [107] measured changes in the index of refraction (IoR) of non-equilibrium, human-blood plasma after a one-minute of TELSAR chip (TC) irradiation via laser fringe patterns. The change in IoR by TC-irradiation was ten-fold larger than that predicted from the laser-heated temperature change, showing that the TC alters the ion-concentration, gradient-driven currents in a therapeutic fashion. The TC changed the IoR of water by shifting the hydrogen-bond angle [107]. Moreover, TC-irradiation changed the infrared spectrum of aqueous, hydrogen peroxide during evaporation. The resultant $\mathrm{O}-\mathrm{H}$ vibrational spectrum was strongly suppressed, implying significant TC-irradiation effects on non-equilibrium, biochemical systems by altering the chemical potential. These tests need replication.

Measurements show variable radioactive-decay rates $( \pm 0.3 \%)$ in ${ }^{3} \mathrm{H},{ }^{22} \mathrm{Na},{ }^{36} \mathrm{Cl},{ }^{44} \mathrm{Ti},{ }^{54} \mathrm{Mn},{ }^{60} \mathrm{Co}$, ${ }^{85} \mathrm{Kr},{ }^{90} \mathrm{Sr},{ }^{108 \mathrm{~m}} \mathrm{Ag},{ }^{133} \mathrm{Ba},{ }^{152} \mathrm{Eu},{ }^{154} \mathrm{Eu},{ }^{222} \mathrm{Rn},{ }^{236} \mathrm{Ra}$, and ${ }^{239} \mathrm{Pu}$ [108-113], and references therein. Periods in the decay rate are: one day, 12.08/year (solar rotation rate), one year, and $\sim 12$ years (sun-spot cycle). Low-energy nuclear theory [114] cannot explain these observations. An improved radioactive-decay theory is needed, for which we propose that EED is appropriate, based on the Stueckelberg Lagrangian density [44] in Equation (8), for which the corresponding Hamiltonian is [57]:

$$
\boldsymbol{H}_{E E D}=\left(\frac{\varepsilon \mathbf{E}^{2}}{2}+\frac{\mathbf{B}^{2}}{2 \mu}\right)+(\rho-\varepsilon \nabla \bullet \mathbf{E}) \Phi-\mathbf{J} \bullet \mathbf{A}+\frac{C^{2}}{2 \mu}+\frac{C \nabla \bullet \mathbf{A}}{\mu} .
$$

The right-most, two terms in Equation (26) modify charged-particle interactions, causing decay-rate variations in proportion to the SLW/SW power (e.g., electron-capture decay and bound electrons/protons in neutron beta decay). One test is change in hydrogen-atom energy levels during SLW/SW irradiation. A second test involves modulation of radioisotope decay rates, as a function of SLW/SW amplitude and frequency. Such tests must be guided by a quantum-EED simulation and will require careful replication.

\section{EED Implications for Quantum Mechanics and General Relativity}

The purpose of this section is to show that QED and general relativity (GR) have fundamental flaws. These flaws include the need for QED renormalization, which is not provable [115]; a zero-radius of convergence for all renormalized power-series expansions in QED [116]; and the inability of QED and GR to explain dark energy, dark matter, and the huge matter/antimatter imbalance in the universe [117]. We then sketch theory programs to address these flaws by replacement of CED with EED.

CED is the basis for QED, which has been called "the 'most correct' scientific theory in history" [118], "the most accurate physical theory ever built" [119], and "the most successful physical theory" [120]. However, Richard Feynman (1965 Nobel Prize in physics) made the following comment on page 128 of his book [115]: "The shell game that we play ... is technically called 'renormalization.' But no matter how clever the word, it is what I would call a dippy process! Having to resort to hocus-pocus has prevented us from proving that the theory of quantum electrodynamics is mathematically self-consistent. ... I suspect that renormalization is not mathematically legitimate." Moreover, Dyson proved that all renormalized power-series, perturbation expansions in QED have zero-radius of convergence [116], making the results meaningless. These examples show the inconsistency and incompleteness of QED (a local field theory), arising from analogous issues in CED (also a local field theory). New physics is needed [121].

QED divergences arise from the local-field (partial-differential) formulation at space-time points. However, all real-world dynamics (measurements) are made over a finite time and a finite spatial domain, corresponding to a spatio-temporal average. Moreover, physicists have been unable to build a "theory of everything." because QED (a local field theory) is fundamentally incompatible with GR (a nonlinear field theory), as noted above. EED is a non-local (and therefore nonlinear) field theory that includes: (a) provable uniqueness; (b) gauge free; (c) full treatment of irrotational (longitudinal) components of $\mathbf{A}, \mathbf{E}$, and $\mathbf{J} ;(\mathrm{d})$ a new scalar field $(C)$; (e) scalar-longitudinal waves whose properties 
are consistent with preliminary experiments [3]; (f) free-space scalar waves; and (g) inclusion of the CED transverse waves. Quantum-EED would then be non-local and gauge free.

We propose a theoretical program for quantum-EED, beginning with topological solutions of CED dynamics [122-136]. The electrodynamic equations yield closed-loop solutions for $\mathbf{E}$ - and $\mathbf{B}$-field lines that are topologically interleaved (analytically and by simulation). Other work formulated a framework [127] for construction of divergence-free, knotted, vector fields, and scalar fields as found in EED. Kedia et al. [137] showed that an initially knotted light field will stay knotted, if and only if the Poynting-vector-flow field is shear free. This work [122-137] provides a firm basis for electrodynamic topological solutions (knots, braids, and Mobius-strips). Furthermore, an explicit analogy exists between CED and the equations for fluid dynamics, as shown (for example) by Martins and Pinheiro [138]. Two recent experimental papers $[139,140]$ showed evidence for knotted vortices (trefoil knots and linked pairs of rings) in fluid-dynamics experiments. Spirichev [141] proposed a version of EED (by analogy to elasticity theory), finding: (i) $A^{\mu}$ is a physically measurable field, as discussed above; (ii) $C$ and the SLW exist; and (iii) PAPF create a particle-antiparticle plasma, which is compressible (elastic), facilitating SLW propagation. Modanese [69-78] has explored non-local, quantum wave-functions via violation of local charge conservation in condensed matter. A high-frequency dipole oscillation at $10 \mathrm{GHz}$ leads to double-retarded integrals [69] from EED; the $\mathbf{E}^{L}$ magnitude then exceeds the $\mathbf{E}^{T}$ magnitude by a factor of $10^{2}-10^{3}$ in the far-field. This theoretical, simulation, and experimental evidence provides a compelling basis for a first-principles, topological approach to EED-quantum and particle physics.

Spergel [142] concluded that general relativity is "... successful in matching observations, but implies the existence of both dark matter and dark energy. These signify that our understanding of physics is incomplete. We will likely need a new idea as profound as general relativity to explain these mysteries ... " Space-time curvature tells masses how to move, and masses tell space-time how to curve. Thus, GR is explicitly nonlinear, and has divergences that cannot be renormalized.

We propose a theoretical program for general-relativistic EED, including the scalar field $(C)$ in the covariant, anti-symmetric, field tensor $\left(F_{\mu \mathrm{v}}\right)$ of Equation (27), which is consistent with Saa [143]. Moreover, the $T^{o o}$ component of the stress-energy tensor becomes $T^{o o}=\left(B^{2} / 2 \mu+C^{2} / 2 \mu+\varepsilon E^{2} / 2\right) / c^{2}$, with $B=|\mathbf{B}|, E=|\mathbf{E}|$, and $C$ as the scalar-field magnitude. Li et al. [144] studied the non-local current density in various nano-devices, as a negative gradient of a scalar function, consistent with EED.

$$
F_{\mu \nu}^{E E D}=\left(\begin{array}{cccc}
C & -\mathrm{E}_{1}(x) / c & -\mathrm{E}_{2}(x) / c & -\mathrm{E}_{3}(x) / c \\
\mathrm{E}_{1}(x) / c & -\mathrm{C} & \mathrm{B}_{3}(x) & -\mathrm{B}_{2}(x) \\
\mathrm{E}_{2}(x) / c & -\mathrm{B}_{3}(x) & -\mathrm{C} & \mathrm{B}_{1}(x) \\
\mathrm{E}_{3}(x) / c & \mathrm{~B}_{2}(x) & -\mathrm{B}_{1}(x) & -\mathrm{C}
\end{array}\right) .
$$

Keller and Hively [82,83] published a GR-extension of EED, showing that only the longitudinal dynamics are changed by EED, allowing elucidation of the SLW and its source. The work [82] is important, because: (i) the $C$ - and $\mathbf{E}^{L}$-fields do not cancel, as in QED but rather form the SLW; (ii) a dispersion relation arises for $\mathbf{E}^{L}$-waves in terms of the Hubble constant $\left(H_{0}\right)$ and the $\mathbf{E}^{L}$ wave-vector amplitude $\left(q_{o}\right)$; and (iii) this approach is a first-principles path to an EED-quantum theory that includes acceleration and gravity. The work [83] is important, because: (iv) $C$ and $\gamma$ (scale factor in Equation (7)) allow consistency between $\mathbf{E}^{L}$ and $A^{\mu}$; (v) $\gamma C$ arises from superposition of longitudinal fields on the energy shell, $|\omega|=c q$; and (vi) free-space SLWs can be emitted by nonlinear, electrodynamic mixing. A quantum-gravity simulation of a spherically-symmetric, lattice with a stationary metric produces polarized regions of positive and negative curvature [71]. These vacuum fluctuations are markedly different from Wheeler's space-time foam.

\section{Conclusions and Prospects}

The above examples show that CED and QED are incomplete. CED omits the irrotational components of $\mathbf{A}, \mathbf{E}$, and $\mathbf{J}$ together with the scalar field (C). EED includes these irrotational (longitudinal) components, providing fresh understanding of scalar-longitudinal waves, energy, and 
momentum. The examples above have the common feature of irrotational currents: (a) seismic precursor signals due to fracturing in the Earth's crust [99], (b) peeling of adhesive tape [102,103], and (c) SLW irradiation by the TESLAR chip [106,107]. These examples generalize to any phenomena involving the breaking/alteration of chemical bonds in inanimate or biological systems and/or irrotational currents (e.g., radial oscillations of solar plasma). The gradient-driven current and the SLW then elucidate currently unexplained electrodynamic phenomena, both laboratory-based [101-107] and astrophysical $[66,100]$.

EED gives the magnetic vector potential ( A) physical reality. Experimental evidence from the VPT [36,37] validates the Maxwell-Lodge effect [34] with $\mathbf{A}$ as a physical field in the classical domain, along with validation by the Aharonov-Bohm effect in the quantum domain. The invention (and experimental validation) of SLW antennas [68] provides a springboard for disruptive technologies in wireless sources of energy generation and power conversion. EED has the potential to produce a revolution in electricity generation and distribution.

Author Contributions: D.R. produced the initial draft; L.M.H. edited the draft and provided additional references, text, and equations. All authors have read and agreed to the published version of the manuscript.

Funding: This research received no external funding.

Conflicts of Interest: The authors declare no conflict of interest.

\section{References}

1. Dyson, F.J. Feynman's proof of the Maxwell equations. Am. J. Phys. 1990, 58, 209-211. [CrossRef]

2. Woodside, D.A. Three-vector and scalar field identities and uniqueness theorems in Euclidean and Minkowski spaces. Am. J. Phys. 2009, 77, 438-446. [CrossRef]

3. Hively, L.M.; Loebl, A.S. Classical and Extended Electrodynamics. Phys. Essays 2019, 32, 112-126. [CrossRef]

4. Giuliani, G. Vector potential, electromagnetic induction and 'physical meaning'. arXiv 2010, arXiv:1005.2350v1. [CrossRef]

5. Barbieri, S.; Cavinato, M.; Giliberti, M. An educational path for the vector potential and it physical implications. arXiv 2013, arXiv:1303.5619v1.

6. Bork, A.M. Maxwell and the vector potential. Isis 1967, 58, 210-222. [CrossRef]

7. Griffiths, D.J. Introduction to Electrodynamics; Prentice-Hall: New Delhi, India, 2007.

8. Jackson, J.D. Classical Electrodynamics; John Wiley \& Sons, Inc.: New York, NY, USA, 1962.

9. Majumdar, P.; Ray, A. Maxwell Electrodynamics in terms of Physical Potentials. Symmetry 2019, 11, 915. [CrossRef]

10. Majumdar, P.; Battacharjee, S. Gauge-free electrodynamics. arXiv 2018, arXiv:0903.4340v4.

11. Nikolova, N.K.; Zimmerman, R.K. Detection of the Time-Dependent Electromagnetic Potential at $1.3 \mathrm{GHz}$, $C E M-R-46$; Department of Electrical and Computer Engineering, McMaster University: Hamilton, ON, Canada, 2007.

12. Konopinski, E.J. What the electromagnetic vector potential describes. Am. J. Phys. 1978, 46, 499-500. [CrossRef]

13. Faraday, M. Experimental Researches in Electricity; Martin, T., Ed.; London 1839-1855; Creative Media Partners LLC: New York, NY, USA, 1839; Volume 1, para. 60-71.

14. Martin, T. (Ed.) Faraday's Diary (1820-1862); G. Bells \& Sons: London, UK, 1932; Volumes 1-7.

15. Jones, H.B. The Life and Letters of Faraday; Cambridge University Press: London, UK, 1870; Volume 2, p. 9.

16. Faraday, M. Experimental researches in electricity. Phil. Trans. R. Soc. London 1852, 142, $25-56$.

17. Faraday, M. On the physical character of the lines of magnetic force. Phil. Mag. 1852, 3, 401-428. [CrossRef]

18. Ehrenberg, W.; Siday, R.E. The refractive index in electron optics and the principles of dynamics. Proc. Phys. Soc. 1949, B62, 8-21. [CrossRef]

19. Aharonov, Y.; Bohm, D. Significance of electromagnetic potentials in the quantum theory. Phys. Rev. 1959, 115, 485-491. [CrossRef] 
20. Tonomura, A.; Osakabe, N.; Matsuda, T.; Kawasaki, T.; Endo, J.; Yano, S.; Yamada, H. Evidence for the Aharonov-Bohm effect with magnetic field completely shielded from electron wave. Phys. Rev. Lett. 1986, 56, 792-795. [CrossRef] [PubMed]

21. Osakabe, N.; Matsuda, T.; Kawasaki, T.; Endo, J.; Tonomura, A.; Yano, S.; Yamada, H. Experimental confirmation of Aharonov-Bohm effect using a toroidal magnetic field confined by a superconductor. Phys. Rev. A 1986, 34, 815-822. [CrossRef] [PubMed]

22. Webb, R.A.; Washburn, S.; Umbach, C.P.; Laibowitz, R.B. Observation of h/e Aharonov-Bohm oscillations in normal-metal rings. Phys. Rev. Lett. 1985, 54, 2696-2699. [CrossRef]

23. Chandrasekhar, V.; Rooks, M.J.; Wind, S.; Prober, D.E. Observation of Aharonov-Bohm electron interference effects with periods $\mathrm{h} / \mathrm{e}$ and $\mathrm{h} / 2 \mathrm{e}$ in individual micron-size, normal-metal rings. Phys. Rev. Lett. 1985, 55, 1610-1613. [CrossRef]

24. Datta, S.; Melloch, M.R.; Bandyopadhyay, S.; Noren, R.; Vaziri, M.; Miller, M.; Reifenberger, R. Novel Interference Effects between Parallel Quantum Wells. Phys. Rev. Lett. 1985, 55, 2344-2347. [CrossRef]

25. Wu, T.T.; Yang, C.N. Concept of nonintegrable phase factors and global formulation of gauge fields. Phys. Rev. D 1975, 12, 3845-3857. [CrossRef]

26. Olofson, A.E.S.; Squire, R.H. Is " $4 n+2$ " a general quantum electromagnetic topological invariant? Int. J. Quantum Chem. 1995, 29, 313-330. [CrossRef]

27. Van Oudenaarden, A.; Devoret, M.H.; Nazarov, Y.V.; Mooij, J.E. Magneto-electric Aharonov-Bohm effect in metal rings. Nature 1998, 391, 768-770. [CrossRef]

28. Varma, R.K. Curl-free vector potential observation on the macroscale for charged particles in a magnetic field compared with that on the micro-scale: The Aharonov-Bohm effect. Phys. Scr. 2012, 86, 045009. [CrossRef]

29. Varma, R.K. Observability of the effects of curl-free magnetic vector potential on the macroscale and the nature of the 'transition amplitude wave'. Pramana J. Phys. 2010, 74, 491-511. [CrossRef]

30. Varma, R.K. From hunches to surprises-Discovering macro-scale quantum phenomena in charged particle dynamics. Curr. Sci. 2012, 103, 497-511.

31. Varma, R.K. Quantum manifestation of systems on the macroscale-The concept of transition state and transition amplitude wave. Pramana J. Phys. 2007, 68, 901-911. [CrossRef]

32. Varma, R.K.; Puntihavelu, A.M.; Banerjee, S.B. Observation of matter wave beat phenomena in the macrodomain for electrons moving along a magnetic field. Phys. Rev. E 2002, 65, 026503. [CrossRef]

33. Shukla, P.K. Curl-free vector potential observed at the macroscale. Phys. Scr. 2012, 86, 048201. [CrossRef]

34. Rousseaux, G.; Kofman, R.; Minazzoli, O. The Maxwell-Lodge effect: Significance of electromagnetic potentials in the classical theory. Eur. Phys. J. D. 2008, 49, 249-256. [CrossRef]

35. Blondel, A. Sur l'énoncé le plus general des lois de l'induction. C. R. Acad. Sci. 1914, 159, 674-679.

36. Daibo, M.; Oshima, S.; Sasaki, Y.; Sugiyama, K. Vector Potential Coil and Transformer. IEEE Trans. Magn. 2013, 51, 100604.

37. Daibo, M.; Oshima, S. Vector Potential Generation Device, Vector Potential Transformer, Shield Permeation Device, Non-Contact Space Electric Field Generation Device, Null Circuit, and Structure for Vector Potential Generation Device. U.S. Patent 10,037,840, 31 July 2018.

38. Maxwell, J.C. Electromagnetic field theory. Trans. Camb. Phil. Soc. 1856, 10, 27-109.

39. Maxwell, J.C. On physical lines of force Parts I-II. Phil. Mag. 1861, 21, 161-346. [CrossRef]

40. Maxwell, J.C. A Dynamical Theory of the Electromagnetic Field, Part III. Phil. Trans. R. Soc. Lond. 1865, 155, 459-512.

41. Einstein, A. Zur Elektrodynamik bewegter Korper. Ann. Phys. 1905, 17, 891-921. [CrossRef]

42. Woodside, D.A. Uniqueness Theorems for classical four-vector fields in Euclidean and Minkowski spaces. J. Math. Phys. 1999, 40, 4911-4943. [CrossRef]

43. Woodside, D.A. Classical four-vector fields in the longitudinal gauge. J. Math. Phys. 2000, 41, 4622-4653. [CrossRef]

44. Stueckelberg, E.C.G. Die Wechselwirkungskräfte in der Elektrodynamik und in der Feldtheorie der Kräfte. Helv. Phys. Acta 1938, 11, 225-244.

45. Liu, L.-X.; Shao, C.-G. Re-estimatation of the upper limit on the photon mass with the solar wind method. Chin. Phys. Lett. 2012, 29, 111401. [CrossRef]

46. Jack, P.M. Physical space as a quaternion structure, I.; Maxwell's equations. A brief note. arXiv 2003, arXiv:math-ph/0307038v1. 
47. Saa, D. An Alternative to Classical Electromagnetic Theory; 2015 ResearchGate Preprint. Available online: https: //d1wqtxts1xzle7.cloudfront.net/37878536/AnAlternativeToClassicalElectromagnetism.pdf?1433987946=\&resp onse-content-disposition=inline\%3B+filename\%3DAn_Alternative_to_Classical_Electromagne.pdf\&Expires $=1608276532 \&$ Signature $=$ As $\sim\{\}$ mC-f71v2-dlaUfmHPyp $\sim\{$ nQ0LSZfFOcDasPZMevUK3LMM6vHIDrCgwUV tonrsLpW-CslZyp29n8aWX9VhZnpubcXWnlUCruAO2QsEjzj9yF8SeiKHu3jSy2NmFhsz9uL0Q0mo7AEo3pG BBgvARg2K1Z9ENyhzkLGwcUp7e8urymbfxbUMuYqK1tVFKXRpO3yvBbnJAHJ1 \{\}9UDbT \{\}8H3iVM76h 3G \{\}DNe-7AAXNQ1lu644Dws \{\}3Q5n6-NXa6HJZzvVb2R9JOw7Ln1bYzbmwlsLrdhTKvD4uLWNTntGziD jfmfh6ZIGELOx0jCL9waEYKtLNnXUC26R0BjRqdpRAPCQ_\&Key-Pair-Id=APKAJLOHF5GGSLRBV4ZA (accessed on 15 December 2020).

48. Saa, D. A Reformulation of Electromagnetism and Relativity Theory; 2019 ResearchGate Preprint. Available online: https://d1wqtxts1xzle7.cloudfront.net/58933563/A_Reformulation_of_Relativity_Theory201 90417-23859-1hnqo2j.pdf?1555516270=\&response-content-disposition=inline\%3B+filename\%3DA_REFO RMULATION_OF_ELECTROMAGNETISM_AND.pdf\&Expires=1608276592\&Signature=a7NLk0dFNWZ 2QgP6YpxWF-QCS \{\}TzMVJrRuOK51pNelw6F78CjOtDftNj0yfiBV4aXyfQSBWQOxdIZoFSjB6NT2ZZL ebAmkwUePFPInyNy7QWqp8B5udaqzP9aPHK-55yaeBHyZxFMVsH7OBEJhnToP2tnrtMe0PkJ5gzk7GZr ta6o6rXpIeIUzWCuA8zMIR \{\}YLSqsH4JiVELlqwE7WabLnwC \{\}ck5z88vA7RQr3ouRsf0nuXj7rXiXnhQ 2Ofa9pJ0STs8p9zL94-T0t-KelCeIPcbBKOpD1BAMFL3PP6qB71m2Le \{\}H6PoYyTfKUi5z84szQntzEA \{\}K PB4N6cIP399Hw_\&Key-Pair-Id=APKAJLOHF5GGSLRBV4ZA (accessed on 15 December 2020).

49. Williamson, J.G. A new linear theory of light and matter. IOP Conf. Ser. J. Phys. Conf. Ser. 2019, 1251, 012050. [CrossRef]

50. Williamson, J.G. Fermions from bosons and the origin of the exclusion principle. Proc. MENDEL 2012. 2012. Available online: https://www.researchgate.net/profile/John_Williamson5/publication/286708466_Fermions_ from_bosons_and_the_origin_of_the_exclusion_principle/links/5e9301f74585150839d69b0b/Fermions-fr om-bosons-and-the-origin-of-the-exclusion-principle.pdf (accessed on 15 December 2020).

51. Williamson, J.G. The nature of the photon and electron. In The Nature of Light: What are Photons VI; Roychoudhuri, C., Kracklauer, A., Raedt, H.D., Eds.; SPIE: Bellingham, WA, USA, 2015.

52. Williamson, J.G.; Van der Mark, M.B. Is the electron a photon with toroidal topology? Ann. Foundation L. Broglie 1997, 22, 133-160.

53. Fock, V.; Podolsky, E. On the quantization of electro-magnetic waves and the interaction of charges. In Dirac Theory; Faddeev, L.D., Khalfin, L.A., Komarov, I.V., Eds.; 1932. Reprinted in Fock, V.A. Selected Work-Quantum Mechanics and Quantum Field Theory; Chapman \& Hall/CRC: New York, NY, USA, 2004; pp. 225-241.

54. Dirac, P.A.M.; Fock, V.A.; Podolsky, B. On Quantum Electrodynamics; Faddeev, L.D., Khalfin, L.A., Komarov, I.V., Eds.; 1932. Reprinted in Fock, V.A. Selected Work-Quantum Mechanics and Quantum Field Theory; Chapman \& Hall/CRC: New York, NY, USA, 2004; pp. 243-255.

55. Ohmura, T. A new formulation on the electromagnetic field. Prog. Theor. Phys. 1956, 16, 684-685. [CrossRef]

56. Milner, S.R. The classical field theory of matter and electricity I: An approach from first principles. Phil. Trans. R. Soc. A Lond. 1960, 253, 185-204.

57. Aharonov, Y.; Bohm, D. Further discussion of the role of electromagnetic potentials in the quantum theory. Phys. Rev. 1963, 130, 1625-1631. [CrossRef]

58. Alicki, R. Generalised electrodynamics. J. Phys. A Math. Gen. 1978, 11, 1807-1813. [CrossRef]

59. Cornille, P. On the propagation of inhomogeneous waves. J. Phys. D App. Phys. 1990, 23, 129-135. [CrossRef]

60. Cornille, P. An electromagnetic wave approach to matter and radiation. J. Electromagn. Waves Appl. 1994, 8, 1425-1442. [CrossRef]

61. Munz, C.-D.; Ones, P.; Schneider, R.; Sonnendrucker, E.; Voss, U. Maxwell's equations when the charge conservation is not satisfied. C. R. Acad. Sci. Paris 1999, 328, 431-436. [CrossRef]

62. Van Vlaenderen, K. Generalization of classical electrodynamics to admit a scalar field and longitudinal waves. Hadron. J. 2001, 24, 609-628.

63. Van Vlaenderen, K.; Waser, A. A generalization of classical electrodynamics for the prediction of scalar field effects. arXiv 2003, arXiv:physics/0305098.

64. Arbab, A.I.; Satti, Z.A. On the generalized Maxwell's equations and their prediction of the electroscalar wave. Prog. Phys. 2009, 2, 8-13. 
65. Arbab, A.I. The modified electromagnetism and the emergent of longitudinal wave. arXiv 2018, arXiv:physics.gen-phys/1403.2687. [CrossRef]

66. Jimenez, J.C.; Maroto, A.L. Cosmological magnetic fields from inflation in extended electromagnetism. Phys. Rev. D 2011, 83, 023514. [CrossRef]

67. Hively, L.M.; Gaikos, G.C. Toward a more complete electrodynamic theory. Int. J. Signals Imaging Syst. Eng. 2012, 5, 3-10. [CrossRef]

68. Hively, L.M. Methods and Apparatus for Generation and Detection of a Scalar Longitudinal Electromagnetic Wave. U.S. Patent 9,306,527, 3 April 2016.

69. Modanese, G. High-frequency electromagnetic emission from non-local wavefunctions. Appl. Sci. 2019, 9, 1982. [CrossRef]

70. Modanese, G. Design for a test for the electromagnetic coupling of non-local wave functions. Results Phys. 2019, 12, 1056-1061. [CrossRef]

71. Modanese, G. Metrics with zero and almost-zero Einstein action in quantum gravity. Symmetry 2019, 11, 1288. [CrossRef]

72. Modanese, G. Covariant formulation of Aharonov-Bohm electrodynamics and its application to coherent tunneling. In Unified Field Mechanics II: Formulations and Empirical Tests; World Scientific Publishing: Singapore, 2018; pp. 268-272.

73. Modanese, G. Time in quantum mechanics and the local non-conservation of the probability current. Mathematics 2018, 6, 155. [CrossRef]

74. Modanese, G. Electromagnetic coupling of strongly non-local quantum mechanics. Phys. B Condens. Matter 2017, 524, 81-84. [CrossRef]

75. Modanese, G. Generalized Maxwell equations and charge conservation censorship. Mod. Phys. Lett. B 2017, 31, 1750052. [CrossRef]

76. Poher, C.; Modanese, G. Enhanced induction into distant coils by YBCO and silicon-graphite electrodes under large current pulses. Phys. Essays 2017, 30, 435-441. [CrossRef]

77. Modanese, G. Oscillating dipole with fractional quantum source in Aharonov-Bohm electrodynamics. Results Phys. 2017, 7, 480-481. [CrossRef]

78. Modanese, G. A New Formulation of Aharonov-Bohm Generalized Electrodynamics. In Proceedings of the 10th International Symposium Honouring Mathematical Physicist Jean-Pierre Vigier, PortoNovo, Italy, 25-28 July 2016; World Scientific Publishing: Singapore, 2016. Available online: https://bia.unibz.it/discovery /fulldisplay/alma991005773028701241/39UBZ_INST:ResearchRepository (accessed on 15 December 2020).

79. Islamov, G.G.; Tomilin, A.K. Spectral problem in a generalized theory of electromagnetic waves. In Proceedings of the 2017 Progress in Electromagnetics Research Symposium-Spring (PIERS), St. Petersburg, Russia, 22-25 May 2017. [CrossRef]

80. Banduric, R. New Electrodynamics; Displacement Field Technologies: Aurora, CO, USA, 2017.

81. Celani, F.; di Tommaso, A.; Vassallo, G. Maxwell's equations and Occam's razor. J. Condens. Matter Nucl. Sci. 2017, 25, 1-29.

82. Keller, O.; Hively, L.M. Electrodynamics in curved space-time: Free space longitudinal wave propagation. Phys. Essays. 2019, 32, 282-291. [CrossRef]

83. Keller, O.; Hively, L.M. Ohmura's extended electrodynamics: Longitudinal aspects in general relativity. J. Phys. Commun. 2019, 3, 115002. [CrossRef]

84. Haralick, R.A. Notes on Generalized Maxwell Equations; Privately published. Available online: https: //emediapress.com/robertharalick/scalar/?hop=robitai (accessed on 15 December 2020).

85. Donev, S.; Tashkova, M. Linear Connection Interpretation of Extended Electrodynamics. In Proceedings of the 11th International Conference Geometry, Integrability and Quantization, Varna, Bulgaria, 5-10 June 2009; Mladenov, I.M., Vilasi, G., Yoshioka, A., Eds.; Avangard Prima Publishing: Sofia, Bulgaria, 2010; pp. 119-126.

86. El-Nabulsi, R.A. On nonlocal complex Maxwell equations and wave motion in electrodynamics and dielectric media. Opt. Quant. Electron. 2018, 50, 170. [CrossRef]

87. Müller, L.; Szabo, R.J. Extended Quantum Field Theory, Index Theory, and the Parity Anomaly. Commun. Math. Phys. 2018, 362, 1049-1109. [CrossRef]

88. Milton, K.A.; Abalo, E.K.; Parashar, P.; Pourtolami, N.; Wagner, J. PT-symmetric quantum electrodynamics and unitarity. Phil. Trans. R. Soc. A 2013, 371, 20120057. [CrossRef] 
89. El-Nabulsi, R.A. Fourth-order Ginzburg-Landau differential equation a la Fisher-Kolmogorov and its implications in superconductivity. Phys. C 2019, 567, 1353545. [CrossRef]

90. Danese, A.E. Advanced Calculus (Vol. I); Allyn \& Bacon Inc.: Boston, MA, USA, 1965.

91. Valone, T. (Ed.) Nikola Tesla's Electricity Unplugged; Adventures Unlimited Press: Kempton, IL, USA, 2013.

92. Monstein, C.; Wesley, J.P. Observation of scalar longitudinal electrodynamic waves. Europhys. Lett. 2002, 59, 514-520. [CrossRef]

93. Meyl, K. Teslastrahlumg—Die drahtlose Ubertragung von Skallerwellen. In Proceedings of the INET Congress Aumenergie-Technologie 2000, Bregenz, Austria, 15-16 April 2000.

94. Okun, L.B. Tests of electric charge conservation and the Pauli Principle. Sov. Phys. Usp. 1989, 32, 543-547. [CrossRef]

95. Agostini, M.; Appel, S.; Bellinik, G.; Benzigero, J.; Bickd, D.; Bonfinij, G.; Bravoq, D.; Caccianigak, B.; Calapricen, F.; Caminata, A.; et al. Test of electric charge conservation with borexino. Phys. Rev. Lett. 2015, 115, 231802. [CrossRef] [PubMed]

96. Jedele, A.; McIntosh, A.B.; Hagel, K.; Huang, M.; Heilborn, L.; Kohley, Z.; May, L.W.; McCleskey, E.; Youngs, M.; Zarrella, A.; et al. Characterizing neutron-proton equilibration in nuclear reactions with subzeptosecond resolution. Phys. Rev. Lett. 2017, 118, 062501. Available online: https: //cyclotron.tamu.edu/progress-reports/2015-2016/2\%20Heavy\%20Ion\%20Reactions/II_37-40_Characterizin g\%20neutron-proton\%20equilibration\%20in\%20nuclear\%20reactions\%20with\%20sub-zeptosecond.pdf (accessed on 15 December 2020). [CrossRef]

97. Jezouin, S.; Iftikhar, Z.; Anthore, A.; Parmentier, F.D.; Gennser, U.; Cavanna, A.; Ouerghi, A.; Levkivskyi, I.P.; Idrisov, E.; Sukhorukov, E.V.; et al. Controlling charge quantization with quantum fluctuations. Nature 2016, 536, 58-62. [CrossRef]

98. Ramo, S.; Whinnery, J.R.; van Duzer, T. Fields and Waves in Communication Electronics; John Wiley \& Sons: New York, NY, USA, 1967; p. 332ff.

99. Park, L. Seismic Activity Detector. U.S. Patent 8,023,360, 29 August 2008.

100. Zaimidoroga, O. An electroscalar energy of the sun. J. Mod. Phys. 2016, 7, 806-818. [CrossRef]

101. Wesley, J.P. Weber electrodynamics: Part I-General theory, steady current effects. Found. Phys. Lett. 1990, 3, 443-469. [CrossRef]

102. Camara, C.G.; Escobar, J.V.; Hird, J.R.; Putterman, S.J. Correlation between nanosecond x-ray flashes and stick-slip friction in peeling tape. Nature 2008, 455, 1089-1092. [CrossRef]

103. Constable, E.; Horvat, J.; Lewis, R.A. Mechanisms of X-ray emission from peeling of adhesive tape. Appl. Phys. Lett. 2010, 97, 131502. [CrossRef]

104. Szabo, I.; Sodderman, M.; Leanza, L.; Zoratti, M.; Gulbins, E. Single-point mutations of a lysine residue change function of Bax and Bcl-xL expressed in Bax- and Bak-less mouse embryonic fibroblasts: Novel insights into the molecular mechanisms of Bax-induced apoptosis. Cell Death Differ. 2011, 18, 427-438. [CrossRef]

105. Peralta, R.G.; Andino, S. Electrical neuroimaging with irrotational sources. Comput. Math. Methods Med. 2015, 801037. [CrossRef]

106. Andreev, E.; Dovbeshko, G.; Krasnoholovets, V. The study of the influence of the TESLAR technology on aqueous solution of some bio-molecules. Res. Lett. Phys. Chem. 2007, 94286. [CrossRef]

107. Krasnoholovets, V.; Tane, J.L. An extended interpretation of the thermodynamic theory including an additional energy associated with a decrease in mass. Int. J. Simul. Model Process 2006, 2, 67-79. [CrossRef]

108. Alburger, D.E.; Harbottle, G.; Norton, E.F. Half-life of 32Si. Earth Planet. Sci. Lett. 1986, 78, 168-176. [CrossRef]

109. Parkhomov, A.G. Deviations from beta radioactivity exponential drop. J. Mod. Phys. 2011, 2, 8635. [CrossRef]

110. Jenkins, J.H.; Fischbach, E.; Javorsek, D., II; Lee, R.H.; Sturrock, P.A. Concerning the time dependence of the decay rate of ${ }^{137}$ Cs. Appl. Radiat. Isot. 2013, 74, 50-55. [CrossRef] [PubMed]

111. O’Keefe, D.; Morreale, B.L.; Lee, R.H.; Buncher, J.B.; Jenkins, J.H.; Fischbach, E.; Gruenwald, T.; Javorsek, D., II; Sturrock, P.A. Spectral content of 22Na/44Ti decay data: Implications for a solar influence. Astrophys. Space Sci. 2013, 344, 297-303. [CrossRef]

112. Mohsinally, T.; Fancher, S.; Czemy, M.; Fischbach, E.; Gruenwald, J.T.; Heim, J.; Jenkins, J.H.; Nistor, J.; $\mathrm{O}^{\prime}$ Keefe, D. Evidence for correlations between fluctuations in ${ }_{54} \mathrm{Mn}$ decay rates and solar storms. Astropart. Phys. 2016, 75, 29-37. [CrossRef] 
113. Sturrock, P.A.; Steinitz, G.; Fischbach, E.; Parkhornov, A.; Scargle, J.D. Analysis of beta-decay data acquired at the Physikalisch-Technische Bundesanstalt: Evidence of a solar influence. Astropart. Phys. 2016, 84, 8-14. [CrossRef]

114. Cook, N.D. Models of the Atomic Nucleus: Unification Through a Lattice of Nucleons, 2nd ed.; Springer: Berlin, Germany, 2010; ISBN 978-3642147364.

115. Feynman, R. QED—The Strange Theory of Light and Matter; Princeton University Press: Princeton, NJ, USA, 1985.

116. Dyson, F.J. Divergence of perturbation theory in quantum electrodynamics. Phys. Rev. 1952, 85, $631-632$. [CrossRef]

117. Morel, L. Determination of the fine structure constant with an accuracy of 81 parts per trillion. Nature 2020, 588, 61-65. [CrossRef]

118. Beautiful Bond. Nature 2017, 547, 257-258.

119. Duplantier, B.; Jolicceur, T. Short range Flaws. Nature 1982, 356, 24. [CrossRef]

120. Bailin, D. Gauge Field Theory. Nature 1976, 260, 736. [CrossRef]

121. Ellis, J. The need for new physics. Nature 2012, 481, 24. [CrossRef] [PubMed]

122. Zilberti, L. The misconception of closed magnetic flux lines. IEEE Magn. Lett. 2017, 8, 1306005. [CrossRef]

123. Arrayas, A.; Bouwmeester, D.; Trueba, J.L. Knots in electromagnetism. Phys. Rep. 2017, 667, 1-61. [CrossRef]

124. Kedia, H.; Foster, D.; Dennis, M.R.; Irvine, W.T.M. Weaving knotted vector fields with tunable helicity. Phys. Rev. Lett. 2016, 117, 274501. [CrossRef]

125. Jhajj, N.; Larkin, I.; Rosenthal, E.W.; Zahedpour, S.; Wahlstrand, J.K.; Milchberg, H.M. Spatiotemporal optical vortices. Phys. Rev. X 2016, 6, 031037. [CrossRef]

126. Kedia, H.; Bialynicki-Birula, I.; Peralta-Salas, D.; Irvine, W.T.M. Tying Knots in Light Fields. Phys. Rev. Lett. 2013, 111, 150404. [CrossRef]

127. Dalhuisen, J.W.; Bouwmeester, D. Twistors and electromagnetic knots. J. Phys. A Math. Theor. 2012, 45, 135201. [CrossRef]

128. Barrio, R.; Blesa, F.; Serrano, S. Topological changes in periodicity hubs of dissipative systems. Phys. Rev. Lett. 2012, 108, 214102. [CrossRef] [PubMed]

129. Goldstein, R.E.; Moffatt, H.K.; Pesci, A.I. Topological constraints and their breakdown in dynamical evolution. Nonlinearity 2012, 25, R85-R98. [CrossRef]

130. Irvine, W.T.M.; Bouwmeester, D. Linked and knotted beams of light. Nature Phys. 2008, 4, 718-720. [CrossRef]

131. Chubykalo, A.E.; Espinoza, A. Unusual formations of the free electromagnetic field in vacuum. J. Phys. A 2002, 35, 8043-8053. [CrossRef]

132. Gilmore, R. Topological analysis of chaotic dynamical systems. Rev. Mod. Phys. 1998, 70, 1455-1529. [CrossRef]

133. Rafiada, A.F.; Trueba, J.L. Two properties of electromagnetic knots. Phys. Lett. A 1997, 232, 25-33.

134. Marsh, G.E. Force-Free Magnetic Fields: Solutions, Topology and Applications; World Scientific Publishing: Singapore, 1996.

135. Ranada, A. A topological theory of the electromagnetic field. Lett. Math. Phys. 1989, 18, 97-106. [CrossRef]

136. McDonald, K.L. Topology of steady current magnetic fields. Am. J. Phys. 1954, 22, 586-596. [CrossRef]

137. Kedia, H.; Peralta-Salas, D.; Irvine, W.T.M. When do knots in light stay knotted? J. Phys. A Math. Theor. 2018, 51, 025204. [CrossRef]

138. Martins, A.A.; Pinheiro, M.J. Fluidic electrodynamics: Approach to electromagnetic propulsion. Phys. Fluids 2009, 21, 097103. [CrossRef]

139. Kleckner, D.; Irvine, W.T.M. Creation and Dynamics of knotted vortices. Nat. Phys. 2013, 9, $253-258$. [CrossRef]

140. Scheeler, M.W.; van Rees, W.M.; Kedia, H.; Kleckner, D.; Irvine, W.T.M. Linking fluids as they twist and writhe. Science 2017, 357, 487-491. [CrossRef] [PubMed]

141. Spirichev, Y.A. About Longitudinal Waves of an Electromagnetic Field. Privately Published. 2017. Available online: https://scholar.google.com/scholar?hl=en\&as_sdt=0\%2C6\&q=\%22About+Longitudinal+ Waves+of+an+Electromagnetic+Field \%22\&btnG= (accessed on 16 December 2020).

142. Spergel, D. The dark side of cosmology: Dark matter and dark energy. Science 2015, 347, 1100-1102. [CrossRef] [PubMed] 
143. Saa, D. General Covariance of Maxwell's Equations. 2019 Preprint (Eq. 3). Available online: https: //www.researchgate.net/publication/332119339 (accessed on 15 December 2020).

144. Li, C.; Wan, L.; Wei, Y.; Wang, J. Definition of current density in the presence of a non-local potential. Nanotechnology 2011, 19, 115412. [CrossRef]

Publisher's Note: MDPI stays neutral with regard to jurisdictional claims in published maps and institutional affiliations.

(C) 2020 by the authors. Licensee MDPI, Basel, Switzerland. This article is an open access article distributed under the terms and conditions of the Creative Commons Attribution (CC BY) license (http://creativecommons.org/licenses/by/4.0/). 